\title{
Subleading power corrections to the pion-photon transition form factor in QCD
}

\author{
Yu-Ming Wang ${ }^{a, b}$ and Yue-Long Shen ${ }^{c}$ \\ ${ }^{a}$ School of Physics, Nankai University, \\ Weijin Road 94, 300071 Tianjin, P.R. China \\ ${ }^{b}$ Fakultät für Physik, Universität Wien, \\ Boltzmanngasse 5, 1090 Vienna, Austria \\ ${ }^{c}$ College of Information Science and Engineering, Ocean University of China, \\ Songling Road 238, Qingdao, 266100 Shandong, P.R. China \\ E-mail: wangyuming@nankai.edu.cn, shenylmeteor@ouc.edu.cn
}

ABSTRACT: We reconsider QCD factorization for the leading power contribution to the $\gamma^{*} \gamma \rightarrow \pi^{0}$ form factor $F_{\gamma^{*} \gamma \rightarrow \pi^{0}}\left(Q^{2}\right)$ at one loop using the evanescent operator approach, and demonstrate the equivalence of the resulting factorization formulae derived with distinct prescriptions of $\gamma_{5}$ in dimensional regularization. Applying the light-cone QCD sum rules (LCSRs) with photon distribution amplitudes (DAs) we further compute the subleading power contribution to the pion-photon form factor induced by the "hadronic" component of the real photon at the next-to-leading-order in $\mathcal{O}\left(\alpha_{s}\right)$, with both naive dimensional regularization and 't Hooft-Veltman schemes of $\gamma_{5}$. Confronting our theoretical predictions of $F_{\gamma^{*} \gamma \rightarrow \pi^{0}}\left(Q^{2}\right)$ with the experimental measurements from the BaBar and the Belle Collaborations implies that a reasonable agreement can be achieved without introducing an "exotic" end-point behaviour for the twist-2 pion DA.

Keywords: Perturbative QCD, Resummation

ARXIV EPRINT: 1706.05680 


\section{Contents}

1 Introduction 1

2 Factorization of the leading power contribution 3

2.1 QCD factorization of $F_{\gamma^{*} \gamma \rightarrow \pi^{0}}\left(Q^{2}\right)$ at tree level 4

2.2 QCD factorization of $F_{\gamma^{*} \gamma \rightarrow \pi^{0}}\left(Q^{2}\right)$ at $\mathcal{O}\left(\alpha_{s}\right) \quad 6$

3 The subleading-power correction from photon DAs 11

$\begin{array}{lll}3.1 & \text { The hadronic photon effect at tree level } & 12\end{array}$

$\begin{array}{ll}3.2 & \text { The hadronic photon effect at one loop } \\ \end{array}$

4 Numerical analysis $\quad 21$

$\begin{array}{lll}4.1 & \text { Theory input parameters } & 21\end{array}$

4.2 Predictions for the $\pi^{0} \gamma^{*} \gamma$ form factor 23

$\begin{array}{llr}5 & \text { Conclusion } & 27\end{array}$

A Two-loop evolution functions $\quad \mathbf{2 8}$

A.1 RG evolution of the twist-2 pion DA at two loops 28

A.2 RG evolution of the twist-2 photon DA at two loops 30

$\begin{array}{ll}\text { B Spectral representations } & 30\end{array}$

\section{Introduction}

Hard exclusive processes play a prominent role in exploring the strong interaction dynamics of hadronic reactions in the framework of QCD. The pion-photon transition form factor $\gamma^{*} \gamma \rightarrow \pi^{0}$ at large momentum transfers $\left(Q^{2}\right)$ serves as one of the simplest exclusive processes for testing the theoretical predictions based upon perturbative QCD factorization. The hard-collinear factorization theorem for the pion-photon form factor $F_{\gamma^{*} \gamma \rightarrow \pi^{0}}\left(Q^{2}\right)$ can be demonstrated at leading power in $1 / Q^{2}$ utilizing both diagrammatic approaches [1-3] and effective field theory techniques [4]. The hard coefficient function entering the leadingtwist factorization formula has been computed at one loop [5-7], and at two loops [8] in the large $\beta_{0}$ approximation. In virtue of the fact that the twist-2 pion distribution amplitude (DA) is defined by an axial-vector light-ray operator, a subtle issue in evaluating QCD corrections to the hard function in dimensional regularization lies in the definition the Dirac matrix $\gamma_{5}$ in the complex $D$-dimensional space demanding a new set of algebraic identities and various prescriptions for the treatment of $\gamma_{5}$ have been proposed to meet the demand of precision QCD calculations in different contexts (see $[9,10]$ for an overview and [11-15] for more discussions). Employing the trace technique, the $\gamma_{5}$ ambiguity of 
dimensional regularization was resolved by adjusting the way of manipulating $\gamma_{5}$ in each diagram to preserve the axial-vector Ward identity [6]. We will demonstrate in this paper the equivalence of factorization formulae for the pion-photon transition form factor constructed with naive dimensional regularization (NDR) and 't Hooft-Veltman (HV) schemes of the $\gamma_{5}$ matrix, using the spinor decomposition technique [16-18] and the evanescent operator approach $[19,20]$.

Confronting the theoretical predictions with the precision experimental measurements of the $\pi^{0} \gamma^{*} \gamma$ form factor at accessible $Q^{2}$ evidently necessities a better understanding of the subleading power terms in the large momentum expansion, due in particular to the scaling violation implied by the BaBar data [21]. The significance of the power suppressed contributions to $F_{\gamma^{*} \gamma \rightarrow \pi^{0}}\left(Q^{2}\right)$ was highlighted by evaluating the soft correction to the leading twist effect with the dispersion approach $[22,23]$ and turned out to be crucial to suppress the contributions from higher Gegenbauer moments of the twist-2 pion DA (see also $[24,25])$. An attractive advantage of the dispersion approach [26] is that the subleading power "hadronic" photon correction is taken into account effectively by modifying the spectral function in the real-photon channel at the price of introducing two nonperturbative parameters (i.e., the vector meson mass $m_{\rho}$ and the effective threshold parameter $s_{0}$ ). This effective method allows continuous improvement of the theoretical accuracy for predicting the pion-photon form factor by including the next-to-next-to-leading order (NNLO) QCD correction to the twist- 2 contribution and the finite-width effect of the unstable vector mesons in the hadronic dispersion relation [27-30]. Further applications of this technique were pursued in radiative leptonic $B$-meson decay [31,32] and electro-production of the pseudoscalar eta mesons [33] and of tensor mesons [34] in an attempt to "overcome" the difficulty of rapidity divergences emerged in the direct QCD calculations of the subleading power contributions. It is then in demand to provide an independent QCD approach to compute the above-mentioned power corrections for the sake of boosting our confidence on the reliability of both theoretical tools. Another objective of this paper is to construct the light-cone sum rules (LCSRs) for the hadronic photon effect in the pion-photon transition form factor with photon distribution amplitudes (DAs) [35] at the next-to-leading order (NLO) in $\alpha_{s}$.

Applying the transverse-momentum-dependent (TMD) factorization scheme for hard exclusive processes [36], the leading power contribution to the pion-photon form factor was also computed at $\mathcal{O}\left(\alpha_{s}\right)$ with the diagrammatic approach [37] (see also [38, 39]), and the joint summation of the parametrically large logarithms $\ln ^{2} \mathbf{k}_{\perp}^{2} / Q^{2}$ and $\ln ^{2} x$ in the hard matching coefficient was performed in Mellin and impact-parameter spaces [25]. However, the subleading power contribution to $F_{\gamma^{*} \gamma \rightarrow \pi^{0}}\left(Q^{2}\right)$ has not been discussed systematically in TMD factorization (see however [40] in the context of the pion electromagnetic form factor). Further development of the TMD factorization for the $\pi^{0} \gamma^{*} \gamma$ form factor with a definite power counting scheme for the intrinsic transverse momentum and of the factorizationcompatible TMD pion wave functions [41] will be essential to put this factorization scheme on a solid ground, albeit with the intensive applications to many hard exclusive processes [42-46]. The dedicated BaBar and Belle measurements [21, 47] of $F_{\gamma^{*} \gamma \rightarrow \pi^{0}}\left(Q^{2}\right)$ also stimulated intensive theoretical investigations with various phenomenological approaches 
as well as lattice QCD simulations (see for instance [48-50]). In particular, an "exotic" twist-two pion DA with the non-vanishing end-point behaviour was proposed $[51,52]$ to accommodate the anomalous BaBar data at high $Q^{2}$, but was soon critically examined in [22] concluding that a reasonable description of the BaBar data in [51, 52] is achieved rather due to the introduction of a sizable nonperturbative soft correction from the TMD pion wavefunction.

The outline of this paper is as follows: in section 2 we recalculate the one-loop hard function entering the factorization formula for the pion-photon form factor at leading power in $1 / Q^{2}$ with both the NDR and HV schemes of $\gamma_{5}$, and demonstrate the renormalizationscheme independence of the factorization formulae for physical quantities explicitly. It will be shown that our expression of the NLO hard-scattering kernel in the NDR scheme reproduces the classical result obtained by Braaten [6] and the renormalization-prescription dependence of the short-distance coefficient at $\mathcal{O}\left(\alpha_{s}\right)$ will be cancelled precisely by the scheme dependent twist-2 pion DA at one loop. We then establish QCD factorization for the vacuum-to-photon correlation function defined with a pseudoscalar interpolating current for the pion state and an electromagnetic current carrying a space-like momentum $q_{\mu}\left(q^{2}=-Q^{2}\right)$ at one loop in section 3. It will be further proved that the resulting hard matching coefficients obtained in the NDR and HV schemes are related by the finite renormalization constant term, which is introduced in the HV scheme in order to fulfill the Adler-Bardeen theorem for the non-renormalization of the axial anomaly [11, 53, 54]. The next-to-leading-logarithmic (NLL) resummation improved LCSR for the hadronic photon correction to $F_{\gamma^{*} \gamma \rightarrow \pi^{0}}\left(Q^{2}\right)$ will be also presented with the aid of the parton-hadron duality ansatz. Taking advantage of the newly derived subleading power correction and the twistfour effect from both the two-particle and three-particle pion DAs at tree level $[22,26]$, we will provide updated theoretical predictions for the pion-photon form factor in section 4 with distinct nonperturbative models for the twist-2 pion DA. A summary of our observations and the concluding remarks are presented in section 5. We collect the two-loop evolution functions for the leading twist DAs of the pion and the photon in appendix A and display the spectral representations of the convolution integrals for the construction of the NLL LCSR of the hadronic photon contribution in appendix B.

\section{Factorization of the leading power contribution}

We will first compute the leading power contribution to the pion-photon form factor at one loop

$$
\left\langle\pi(p)\left|j_{\mu}^{\mathrm{em}}\right| \gamma\left(p^{\prime}\right)\right\rangle=g_{\mathrm{em}}^{2} \epsilon_{\mu \nu \alpha \beta} q^{\alpha} p^{\beta} \epsilon^{\nu}\left(p^{\prime}\right) F_{\gamma^{*} \gamma \rightarrow \pi^{0}}\left(Q^{2}\right),
$$

with both the NDR and HV schemes for the $\gamma_{5}$ matrix in $D$ dimensions, applying the technique of soft-collinear effective theory (SCET) and the evanescent operator approach. The main purpose of this section is to provide an alternative way to establish the QCD factorization theorem for the leading twist contribution to the $\pi^{0} \gamma^{*} \gamma$ form factor at the operator level. Here, $q=p-p^{\prime}, p$ refers to the four-momentum of the pion, the on-shell 


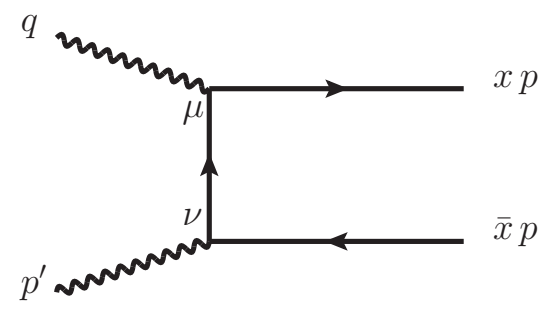

(a)

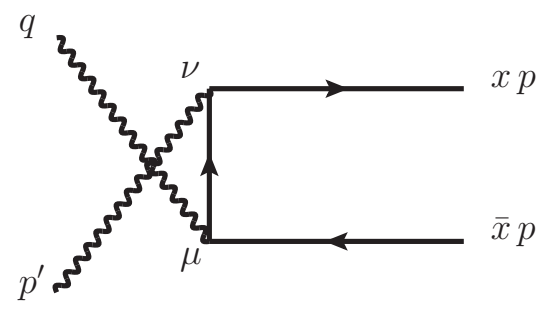

(b)

Figure 1. Diagrammatical representation of the tree-level contribution to the partonic amplitude $\gamma \gamma^{*} \rightarrow q \bar{q}$ induced by two electromagnetic currents.

photon carries the four-momentum $p^{\prime}$ and

$$
j_{\mu}^{\mathrm{em}}=\sum_{q} g_{\mathrm{em}} Q_{q} \bar{q} \gamma_{\mu} q, \quad \epsilon_{0123}=-1 .
$$

We further introduce a light-cone vector $\bar{n}_{\mu}$ parallel to the photon momentum $p^{\prime}$, define another light-cone vector $n_{\mu}$ along the direction of the momentum $p$ in the massless pion limit, and employ the following power counting scheme at large momentum transfer

$$
\bar{n} \cdot p \sim n \cdot p^{\prime} \sim \mathcal{O}\left(\sqrt{Q^{2}}\right), \quad n \cdot p \sim \mathcal{O}\left(\Lambda^{2} / \sqrt{Q^{2}}\right) .
$$

\subsection{QCD factorization of $F_{\gamma^{*} \gamma \rightarrow \pi^{0}}\left(Q^{2}\right)$ at tree level}

QCD factorization for the leading-twist contribution to the $\gamma^{*} \gamma \rightarrow \pi^{0}$ form factor at tree level can be established by inspecting the four-point QCD matrix element

$$
\Pi_{\mu}=\left\langle q(x p) \bar{q}(\bar{x} p)\left|j_{\mu}^{\mathrm{em}}\right| \gamma\left(p^{\prime}\right)\right\rangle
$$

at leading order (LO) in $\alpha_{s}$, where $x$ indicates the momentum fraction carried by the collinear quark of the pion and $\bar{x} \equiv 1-x$. Computing the two diagrams displayed in figure 1 yields

$$
\begin{aligned}
\Pi_{\mu}^{(0)} & =-\frac{i g_{\mathrm{em}}^{2}\left(Q_{u}^{2}-Q_{d}^{2}\right)}{2 \sqrt{2} \bar{n} \cdot p} \epsilon^{\nu}\left(p^{\prime}\right)\left\{\frac{\left[\bar{u}(x p) \gamma_{\mu, \perp} \not \not h \gamma_{\nu, \perp} v(\bar{x} p)\right]}{\bar{x}}-\frac{\left[\bar{u}(x p) \gamma_{\nu, \perp} \not h \gamma_{\mu, \perp} v(\bar{x} p)\right]}{x}\right\} \\
& =-\frac{i g_{\mathrm{em}}^{2}\left(Q_{u}^{2}-Q_{d}^{2}\right)}{2 \sqrt{2} \bar{n} \cdot p} \epsilon^{\nu}\left(p^{\prime}\right)\left[\frac{1}{\bar{x}^{\prime}} *\left\langle O_{A, \mu \nu}\left(x, x^{\prime}\right)\right\rangle^{(0)}-\frac{1}{x^{\prime}} *\left\langle O_{B, \mu \nu}\left(x, x^{\prime}\right)\right\rangle^{(0)}\right] .
\end{aligned}
$$

Here, $\left\langle O_{A, \mu \nu}\right\rangle^{(0)}$ and $\left\langle O_{B, \mu \nu}\right\rangle^{(0)}$ denote the tree-level partonic matrix elements of the collinear operators $O_{A, \mu \nu}$ and $O_{B, \mu \nu}$ in SCET

$$
\left\langle O_{j, \mu \nu}\left(x, x^{\prime}\right)\right\rangle \equiv\left\langle q(x p) \bar{q}(\bar{x} p)\left|O_{j, \mu \nu}\left(x^{\prime}\right)\right| 0\right\rangle=\bar{\xi}(x p) \Gamma_{j, \mu \nu} \xi(\bar{x} p) \delta\left(x-x^{\prime}\right)+\mathcal{O}\left(\alpha_{s}\right),
$$

and the convolution integration is represented by an asterisk. The definition of the SCET operator $O_{j, \mu \nu}$ in the momentum space is given by

$$
O_{j, \mu \nu}\left(x^{\prime}\right)=\frac{\bar{n} \cdot p}{2 \pi} \int d \tau e^{-i x^{\prime} \tau \bar{n} \cdot p} \bar{\xi}(\tau \bar{n}) W_{c}(\tau \bar{n}, 0) \Gamma_{j, \mu \nu} \xi(0),
$$


with the collinear Wilson line

$$
W_{c}(\tau \bar{n}, 0)=\mathrm{P}\left\{\operatorname{Exp}\left[i g_{s} \int_{0}^{\tau} d \lambda \bar{n} \cdot A_{c}(\lambda \bar{n})\right]\right\}
$$

and

$$
\Gamma_{A, \mu \nu}=\gamma_{\mu, \perp} \not h \gamma_{\nu, \perp}, \quad \Gamma_{B, \mu \nu}=\gamma_{\nu, \perp} \not \not h \gamma_{\mu, \perp} .
$$

To facilitate the determination of the hard function entering the leading power factorization formula of $F_{\gamma^{*} \gamma \rightarrow \pi^{0}}\left(Q^{2}\right)$, we employ the SCET operator basis $\left\{O_{1, \mu \nu}, O_{2, \mu \nu}, O_{E, \mu \nu}\right\}$ with

$$
\Gamma_{1, \mu \nu}=g_{\mu \nu}^{\perp} \not h, \quad \Gamma_{2, \mu \nu}=i \epsilon_{\mu \nu}^{\perp} \not h \gamma_{5}, \quad \Gamma_{E, \mu \nu}=\not h\left(\frac{\left[\gamma_{\mu, \perp}, \gamma_{\nu, \perp}\right]}{2}-i \epsilon_{\mu \nu}^{\perp} \gamma_{5}\right),
$$

where $O_{E, \mu \nu}$ is an evanescent operator vanishing in four dimensions and

$$
g_{\mu \nu}^{\perp} \equiv g_{\mu \nu}-\frac{n_{\mu} \bar{n}_{\nu}}{2}-\frac{n_{\nu} \bar{n}_{\mu}}{2}, \quad \epsilon_{\mu \nu}^{\perp} \equiv \frac{1}{2} \epsilon_{\mu \nu \alpha \beta} \bar{n}^{\alpha} n^{\beta} .
$$

It needs to be pointed out that the $\epsilon$-tensor is always defined in four dimensions, since its indices will be never contracted with a $D$-dimensional index. The effective operator $O_{1, \mu \nu}$ cannot couple with a collinear pion state due to the parity conservation. Taking advantages of the operator identities

$$
\begin{aligned}
& O_{A, \mu \nu}=-\left(O_{1, \mu \nu}+O_{2, \mu \nu}+O_{E, \mu \nu}\right), \\
& O_{B, \mu \nu}=-\left(O_{1, \mu \nu}-O_{2, \mu \nu}-O_{E, \mu \nu}\right),
\end{aligned}
$$

we observe that the two tree-level diagrams in figure 1 give rise to the identical contribution to the pion-photon transition form factor and such observation can be further generalized to all orders in QCD applying the charge-conjugation transformation.

We now employ the operator matching equation with the evanescent operator

$$
\Pi_{\mu}=\left[\frac{i g_{\mathrm{em}}^{2}\left(Q_{u}^{2}-Q_{d}^{2}\right)}{2 \sqrt{2} \bar{n} \cdot p} \epsilon^{\nu}\left(p^{\prime}\right)\right] \sum_{i} T_{i}\left(x^{\prime}\right) *\left\langle O_{i, \mu \nu}\left(x, x^{\prime}\right)\right\rangle,
$$

and expand all quantities to the tree level, yielding

$$
T_{1}^{(0)}\left(x^{\prime}\right)=\frac{1}{x^{\prime}}-\frac{1}{\bar{x}^{\prime}}, \quad T_{2}^{(0)}\left(x^{\prime}\right)=T_{E}^{(0)}\left(x^{\prime}\right)=\frac{1}{x^{\prime}}+\frac{1}{\bar{x}^{\prime}} .
$$

Utilizing the definition of the leading twist pion DA on the light cone

$$
\left\langle\pi(p)\left|\bar{\xi}(y) W_{c}(y, 0) \gamma_{\mu} \gamma_{5} \xi(0)\right| 0\right\rangle=-i f_{\pi} p_{\mu} \int_{0}^{1} d u e^{i u p \cdot y} \phi_{\pi}(u, \mu)+\mathcal{O}\left(y^{2}\right),
$$

it is straightforward to derive the tree-level factorization formula of the $\pi^{0} \gamma^{*} \gamma$ form factor

$$
F_{\gamma^{*} \gamma \rightarrow \pi^{0}}^{\mathrm{LP}}\left(Q^{2}\right)=\frac{\sqrt{2}\left(Q_{u}^{2}-Q_{d}^{2}\right) f_{\pi}}{Q^{2}} \int_{0}^{1} d x T_{2}^{(0)}(x) \phi_{\pi}(x, \mu)+\mathcal{O}\left(\alpha_{s}\right) .
$$




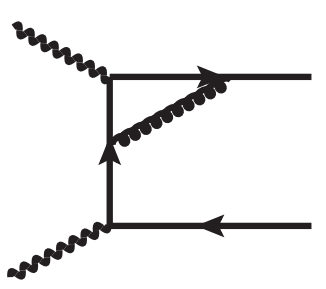

(a)

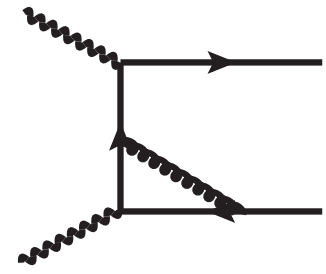

(b)

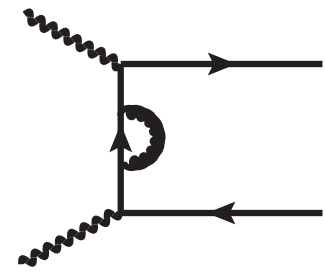

(c)

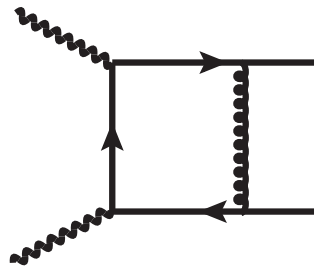

(d)

Figure 2. Diagrammatical representation of the one-loop contribution to the partonic amplitude $\gamma \gamma^{*} \rightarrow q \bar{q}$ induced by two electromagnetic currents. The corresponding symmetric diagrams obtained by exchanging the two photon states are not shown.

\subsection{QCD factorization of $F_{\gamma^{*} \gamma \rightarrow \pi^{0}}\left(Q^{2}\right)$ at $\mathcal{O}\left(\alpha_{s}\right)$}

We proceed to compute the NLO QCD correction to the four-point partonic amplitude $\Pi_{\mu}^{(1)}$ at leading power in $1 / Q^{2}$ for the determination of the hard function $T_{2}$ at $\mathcal{O}\left(\alpha_{s}\right)$. It needs to be stressed that the QCD matrix element $\Pi_{\mu}$ defined by two electromagnetic currents is independent of the prescription of $\gamma_{5}$ in the $D$-dimensional space and the renormalization scheme dependence of the perturbative matching coefficient $T_{2}$ comes solely from the radiative correction to the twist-2 pion DA $\phi_{\pi}(x, \mu)$, whose definition depends on the precise treatment of the Dirac matrix $\gamma_{5}$ in dimensional regularization, due to the infrared subtraction. Evaluating the hard contribution to the one-loop diagrams displayed in figure 2 with the method of regions [55] leads to

$$
\begin{aligned}
\Pi_{\mu}^{(1 a)}= & \frac{i g_{\mathrm{em}}^{2}\left(Q_{u}^{2}-Q_{d}^{2}\right)}{2 \sqrt{2} \bar{n} \cdot p} \frac{\alpha_{s} C_{F}}{2 \pi} \epsilon^{\nu}\left(p^{\prime}\right)\left\langle O_{2, \mu \nu}\left(x, x^{\prime}\right)\right\rangle^{(0)} \\
& *\left\{\frac{1}{x^{\prime} \bar{x}^{\prime}}\left[-\left(\ln \bar{x}^{\prime}+\frac{x^{\prime}}{2}\right)\left(\frac{1}{\epsilon}+\ln \frac{\mu^{2}}{Q^{2}}\right)+\frac{1}{2} \ln \bar{x}^{\prime}\left(\ln \bar{x}^{\prime}-2-\bar{x}^{\prime}\right)-2 x^{\prime}\right]+\ldots\right. \\
\Pi_{\mu}^{(1 b)}= & \frac{i g_{\mathrm{em}}^{2}\left(Q_{u}^{2}-Q_{d}^{2}\right)}{2 \sqrt{2} \bar{n} \cdot p} \frac{\alpha_{s} C_{F}}{2 \pi} \epsilon^{\nu}\left(p^{\prime}\right)\left\langle O_{2, \mu \nu}\left(x, x^{\prime}\right)\right\rangle^{(0)} \\
& *\left\{\frac{1}{\bar{x}^{\prime}}\left[-\frac{1}{2}\left(\frac{1}{\epsilon}+\ln \frac{\mu^{2}}{Q^{2}}-\ln \bar{x}^{\prime}\right)-2\right]\right\}+\ldots, \\
\Pi_{\mu}^{(1 c)}= & -\frac{i g_{\mathrm{em}}^{2}\left(Q_{u}^{2}-Q_{d}^{2}\right)}{2 \sqrt{2} \bar{n} \cdot p} \frac{\alpha_{s} C_{F}}{4 \pi} \epsilon^{\nu}\left(p^{\prime}\right)\left\langle O_{2, \mu \nu}\left(x, x^{\prime}\right)\right\rangle^{(0)} *\left\{\frac{1}{\bar{x}^{\prime}}\left[\frac{1}{\epsilon}+\ln \frac{\mu^{2}}{\bar{x}^{\prime} Q^{2}}+1\right]\right\}+\ldots, \\
\Pi_{\mu}^{(1 d)}= & \frac{i g_{\mathrm{em}}^{2}\left(Q_{u}^{2}-Q_{d}^{2}\right)}{2 \sqrt{2} \bar{n} \cdot p} \frac{\alpha_{s} C_{F}}{2 \pi} \epsilon^{\nu}\left(p^{\prime}\right)\left\langle O_{2, \mu \nu}\left(x, x^{\prime}\right)\right\rangle^{(0)} \\
& *\left\{\frac{\left.\ln \bar{x}^{\prime}\left[\frac{1}{x^{\prime}}+\ln \frac{\mu^{2}}{Q^{2}}-\frac{1}{2} \ln \bar{x}^{\prime}+5\right]\right\}+\ldots,}{}\right.
\end{aligned}
$$

where the ellipses represent terms proportional to $\left\langle O_{1, \mu \nu}\left(x, x^{\prime}\right)\right\rangle^{(0)}$ and $\left\langle O_{E, \mu \nu}\left(x, x^{\prime}\right)\right\rangle^{(0)}$. Adding up different pieces together we can readily obtain the QCD matrix element $\Pi_{\mu}$ at 
$\mathcal{O}\left(\alpha_{s}\right)$

$$
\Pi_{\mu}^{(1)}=\frac{i g_{\mathrm{em}}^{2}\left(Q_{u}^{2}-Q_{d}^{2}\right)}{2 \sqrt{2} \bar{n} \cdot p} \epsilon^{\nu}\left(p^{\prime}\right)\left\langle O_{2, \mu \nu}\left(x, x^{\prime}\right)\right\rangle^{(0)} * A_{2, \text { hard }}^{(1)}\left(x^{\prime}\right)+\ldots
$$

where the $\gamma_{5}$-prescription independent amplitude $A_{2 \text {,hard }}^{(1)}$ reads

$$
\begin{aligned}
A_{2, \text { hard }}^{(1)}\left(x^{\prime}\right)=\frac{\alpha_{s} C_{F}}{4 \pi}\{ & \frac{1}{\bar{x}^{\prime}}\left[-\left(2 \ln \bar{x}^{\prime}+3\right)\left(\frac{1}{\epsilon}+\ln \frac{\mu^{2}}{Q^{2}}\right)+\ln ^{2} \bar{x}^{\prime}+7 \frac{\bar{x}^{\prime} \ln \bar{x}^{\prime}}{x^{\prime}}-9\right] \\
& \left.+\left(x^{\prime} \leftrightarrow \bar{x}^{\prime}\right)\right\} .
\end{aligned}
$$

Expanding the matching equation (2.13) to the one-loop order yields

$$
\begin{aligned}
& {\left[\frac{i g_{\mathrm{em}}^{2}\left(Q_{u}^{2}-Q_{d}^{2}\right)}{2 \sqrt{2} \bar{n} \cdot p} \epsilon^{\nu}\left(p^{\prime}\right)\right] \sum_{i} A_{i}^{(1)}\left(x^{\prime}\right) *\left\langle O_{i, \mu \nu}\left(x, x^{\prime}\right)\right\rangle^{(0)}} \\
& =\left[\frac{i g_{\mathrm{em}}^{2}\left(Q_{u}^{2}-Q_{d}^{2}\right)}{2 \sqrt{2} \bar{n} \cdot p} \epsilon^{\nu}\left(p^{\prime}\right)\right] \sum_{i}\left[T_{i}^{(1)}\left(x^{\prime}\right) *\left\langle O_{i, \mu \nu}\left(x, x^{\prime}\right)\right\rangle^{(0)}+T_{i}^{(0)}\left(x^{\prime}\right) *\left\langle O_{i, \mu \nu}\left(x, x^{\prime}\right)\right\rangle^{(1)}\right] .
\end{aligned}
$$

Now we are in a position to derive the master formula for the one-loop perturbative matching coefficient $T_{i}^{(1)}$ by implementing both the ultraviolet (UV) renormalization and the infrared (IR) subtraction. Following the strategy presented in [18] the UV renormalized matrix element of the SCET operator $O_{i, \mu \nu}$ at $\mathcal{O}\left(\alpha_{s}\right)$ is given by

$$
\left\langle O_{i, \mu \nu}\right\rangle^{(1)}=\sum_{j}\left[M_{i j, \mathrm{bare}}^{(1) R}+Z_{i j}^{(1)}\right] *\left\langle O_{j, \mu \nu}\right\rangle^{(0)},
$$

where the bare matrix element $M_{i j, \text { bare }}^{(1)}$ depends on the IR regularization scheme $R$. Applying the dimensional regularization for both the UV and IR divergences, the bare matrix element $M_{i j, \text { bare }}^{(1)}$ vanishes due to scaleless integrals entering the relevant one-loop computation. Inserting (2.24) into (2.23) and comparing the coefficient of $\left\langle O_{2, \mu \nu}\right\rangle^{(0)}$ give rise to

$$
T_{2}^{(1)}=A_{2}^{(1)}-\sum_{i=1,2, E} T_{i}^{(0)} * Z_{i 2}^{(1)} .
$$

We then need to evaluate the above-mentioned renormalization constants $Z_{i 2}^{(1)}$ individually. Computing the one-loop SCET matrix element $\left\langle O_{1, \mu \nu}\right\rangle^{(1)}$ leads to

$$
\left\langle O_{1, \mu \nu}\right\rangle^{(1)}=Z_{11}\left\langle O_{1, \mu \nu}\right\rangle^{(0)},
$$

indicating the absence of the operator mixing between $O_{1 \mu \nu}$ and $O_{2 \mu \nu}$ under renormalization. The renormalization constant $Z_{11}$ governs the renormalization-scale evolution of the leading twist (longitudinally polarized) $\rho$-meson DA. In addition, the IR subtraction term $T_{2}^{(0)} * Z_{22}^{(1)}$ will remove the collinear contribution to the QCD amplitude $\Pi_{\mu}$ at one loop so that the matching coefficient $T_{2}^{(1)}$ only encodes the information of strong interaction dynamics at the hard scale. Technically, the collinear subtraction has been automatically implemented in the above computation of the QCD matrix element $\Pi_{\mu}$, since only the 
hard contribution computed with the expansion by regions enters the expression of $A_{2 \text {,hard }}^{(1)}$ displayed in (2.22).

We are now ready to discuss the renormalization constant $Z_{E 2}$ of the evanescent operator $O_{E, \mu \nu}$ for the derivation of the final result of the matching coefficient $T_{2}^{(1)}$. Applying the renormalization prescription that the IR finite matrix element of the evanescent operator $\left\langle O_{E, \mu \nu}\right\rangle$ vanishes with dimensional regularization applied only to the UV divergences and with the IR singularities regularized by any parameter other than the dimensions of spacetime $[19,20]$ and making use of the identity (2.24) yield

$$
Z_{E 2}^{(1)}=-M_{E 2}^{(1) \mathrm{off}} \text {. }
$$

The one-loop matching coefficient of the physical operator $O_{2, \mu \nu}$ can be readily obtained by substituting (2.27) into (2.25)

$$
T_{2}^{(1)}=A_{2}^{(1)}-T_{2}^{(0)} * Z_{22}^{(1)}+T_{E}^{(0)} * M_{E 2}^{(1) \text { off }}=A_{2, \text { hard }}^{(1)}+T_{E}^{(0)} * M_{E 2}^{(1) \text { off }} .
$$

The one-loop contribution to the matrix element of the evanescent operator $O_{E, \mu \nu}$ depends on the renormalization prescription of $\gamma_{5}$ in the $D$-dimensional space. We will employ both the NDR and HV schemes of $\gamma_{5}$ below for the illustration of the prescription independence of the factorization formula of $F_{\gamma^{*} \gamma \rightarrow \pi^{0}}\left(Q^{2}\right)$ at $\mathcal{O}\left(\alpha_{s}\right)$ and at leading power in $1 / Q^{2}$. This amounts to showing that the renormalization scheme dependence of the short-distance coefficient function cancels against that of the twist-2 pion DA precisely. Evaluating the SCET diagrams displayed in figure 3 with the Wilson-line Feynman rules, we find that only a single diagram $3\left(\right.$ a) can generate a nonvanishing contribution to $M_{E 2}^{(1) \text { off }}$ using the NDR scheme of $\gamma_{5}$, which turns out to be proportional to the spin-dependent term of the Brodsky-Lepage evolution kernel $[1,2]$. The resulting expression of the infrared subtraction term $T_{E}^{(0)} * M_{E 2}^{(1) \text { off }}$ is given by

$$
\begin{aligned}
\left.T_{E}^{(0)} * M_{E 2}^{(1) \text { off }}\right|_{\mathrm{NDR}} & =\frac{\alpha_{s} C_{F}}{2 \pi} \int_{0}^{1} d y\left(\frac{1}{y}+\frac{1}{\bar{y}}\right) 4\left[\frac{\bar{y}}{\bar{x}^{\prime}} \theta\left(y-x^{\prime}\right)+\frac{y}{x^{\prime}} \theta\left(x^{\prime}-y\right)\right] \\
& =\frac{\alpha_{s} C_{F}}{2 \pi}(-4)\left(\frac{\ln \bar{x}^{\prime}}{x^{\prime}}+\frac{\ln x^{\prime}}{\bar{x}^{\prime}}\right) .
\end{aligned}
$$

However, computing the one-loop matrix element of the evanescent operator $O_{E, \mu \nu}$ with the HV scheme of $\gamma_{5}$ leads to

$$
\left.T_{E}^{(0)} * M_{E 2}^{(1) \mathrm{off}}\right|_{\mathrm{HV}}=0 .
$$

Inserting (2.22), (2.29) and (2.30) into the master formula (2.28), we obtain

$T_{2}^{(1)}\left(x^{\prime}, \mu\right)=\frac{\alpha_{s} C_{F}}{4 \pi}\left\{\frac{1}{\bar{x}^{\prime}}\left[-\left(2 \ln \bar{x}^{\prime}+3\right) \ln \frac{\mu^{2}}{Q^{2}}+\ln ^{2} \bar{x}^{\prime}+\delta \frac{\bar{x}^{\prime} \ln \bar{x}^{\prime}}{x^{\prime}}-9\right]+\left(x^{\prime} \leftrightarrow \bar{x}^{\prime}\right)\right\}$,

where the renormalization scheme dependent parameter $\delta$ is given by

$$
\delta= \begin{cases}-1, & {[\text { NDR scheme }]} \\ +7 . & {[\text { HV scheme }]}\end{cases}
$$




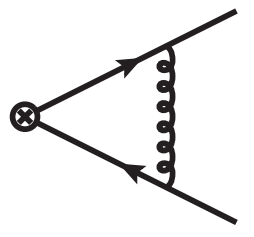

(a)

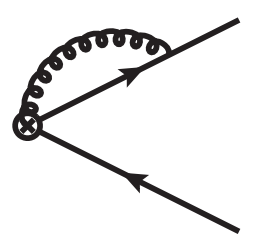

(b)

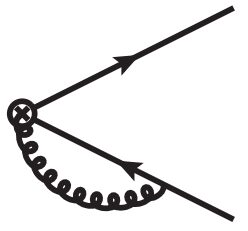

(c)

Figure 3. The one-loop SCET diagrams contributing to $M_{E 2}^{(1) \text { off }}$ and the vertex " $\otimes$ " indicating an insertion of the evanescent operator $O_{E, \mu \nu}$.

Our result of $T_{2}^{(1)}$ in the NDR scheme is identical to that presented in [6] using the trace formalism.

We now aim at demonstrating the renormalization prescription independence of the one-loop factorization formula for the pion-photon form factor

$$
F_{\gamma^{*} \gamma \rightarrow \pi^{0}}^{\mathrm{LP}}\left(Q^{2}\right)=\frac{\sqrt{2}\left(Q_{u}^{2}-Q_{d}^{2}\right) f_{\pi}}{Q^{2}} \int_{0}^{1} d x\left[T_{2}^{(0)}(x)+T_{2}^{(1), \Delta}(x, \mu)\right] \phi_{\pi}^{\Delta}(x, \mu)+\mathcal{O}\left(\alpha_{s}^{2}\right),
$$

where the superscript " $\Delta$ " indicates the $\gamma_{5}$-scheme in dimensional regularization. Taking advantage of the relation of the twist-2 pion DA between the NDR and HV schemes

$$
\phi_{\pi}^{\mathrm{HV}}(x, \mu)=\int_{0}^{1} d y Z_{\mathrm{HV}}^{-1}(x, y, \mu) \phi_{\pi}^{\mathrm{NDR}}(y, \mu),
$$

where the finite renormalization kernel $Z_{\mathrm{HV}}^{-1}$ is given by [56]

$$
Z_{\mathrm{HV}}^{-1}(x, y, \mu)=\delta(x-y)+\frac{\alpha_{s} C_{F}}{2 \pi} 4\left[\frac{x}{y} \theta(y-x)+\frac{\bar{x}}{\bar{y}} \theta(x-y)\right]+\mathcal{O}\left(\alpha_{s}^{2}\right) .
$$

It is then straightforward to show that

$$
\begin{aligned}
\int_{0}^{1} d x T_{2}^{(0)}(x)\left[\phi_{\pi}^{\mathrm{HV}}(x, \mu)-\phi_{\pi}^{\mathrm{NDR}}(x, \mu)\right] \\
\quad=\frac{\alpha_{s} C_{F}}{2 \pi}(-4) \int_{0}^{1} d y\left(\frac{\ln \bar{y}}{y}+\frac{\ln y}{\bar{y}}\right) \phi_{\pi}^{\mathrm{NDR}}(x, \mu)+\mathcal{O}\left(\alpha_{s}^{2}\right),
\end{aligned}
$$

which cancels against the renormalization scheme dependence of the NLO hard function $T_{2}^{(1), \Delta}(x, \mu)$ as displayed in (2.31). We emphasize again that the $\gamma_{5}$-prescription independence of the leading power factorization formula for $F_{\gamma^{*} \gamma \rightarrow \pi^{0}}\left(Q^{2}\right)$ stems from the fact that the QCD matrix element $\left\langle q(x p) \bar{q}(\bar{x} p)\left|j_{\mu}^{\mathrm{em}}\right| \gamma\left(p^{\prime}\right)\right\rangle$ itself is free of the $\gamma_{5}$ ambiguity in dimensional regularization and both the NDR and HV prescriptions can be employed to construct QCD factorization theorems for hard processes provided that the corresponding matching coefficients are computed in an appropriate way without overlooking the potential evanescent operators.

The renormalization scale independence of the factorization formula (2.33) can be readily verified by making use of the evolution equation of the pion DA $\phi_{\pi}(x, \mu)$

$$
\mu^{2} \frac{d}{d \mu^{2}} \phi_{\pi}(x, \mu)=\int_{0}^{1} d y V(x, y) \phi_{\pi}(y, \mu),
$$


where the evolution kernel $V(x, y)$ can be expanded perturbatively in QCD

$$
V(x, y)=\sum_{n=0}\left(\frac{\alpha_{s}}{4 \pi}\right)^{n+1}\left[V_{n}(x, y)\right]_{+},
$$

with the "plus" function defined as

$$
[f(x, y)]_{+}=f(x, y)-\delta(x-y) \int_{0}^{1} d t f(t, y)
$$

and the LO Brodsky-Lepage kernel given by $[1,2]$

$$
V_{0}(x, y)=2 C_{F}\left[\frac{1-x}{1-y}\left(1+\frac{1}{x-y}\right) \theta(x-y)+\frac{x}{y}\left(1+\frac{1}{y-x}\right) \theta(y-x)\right] .
$$

It is appropriate to point out that the one-loop evolution kernel $V_{0}(x, y)$ is independent of the $\gamma_{5}$ prescription in the complex $D$-dimensional space, however, the two-loop evolution kernel $V_{1}(x, y)$ does depend on the renormalization scheme. Applying the renormalizationgroup (RG) equation (2.37) then leads to

$$
\frac{d}{d \ln \mu} F_{\gamma^{*} \gamma \rightarrow \pi^{0}}\left(Q^{2}\right)=\mathcal{O}\left(\alpha_{s}^{2}\right) .
$$

We further turn to sum the parametrically large logarithms of $Q^{2} / \mu^{2}$ in the shortdistance function at next-to-leading-logarithmic (NLL) accuracy employing the standard RG approach in the momentum space. Technically, the desired NLL resummation can be readily achieved by setting the factorization scale of order $\mu \sim \sqrt{Q^{2}}$ and evolving the leading twist pion DA up to that scale at two loops. The NLO evolution kernel $V_{1}(x, y)$ was first obtained with the diagrammatic approach in the light-cone gauge [57, 58], then in the Feynman gauge [59, 60], and was further reconstructed [61] based upon the knowledge of the conformal anomalies and the available forward DGLAP splitting functions at $\mathcal{O}\left(\alpha_{s}^{2}\right)$. The two-loop evolution potential $V_{1}(x, y)$ can be organized as

$$
V_{1}(x, y)=2 N_{f} C_{F} V_{N}(x, y)+2 C_{F} C_{A} V_{G}(x, y)+C_{F}^{2} V_{F}(x, y),
$$

where $N_{f}$ is the number of the active quark flavours. The explicit expressions of the kernels $V_{N}, V_{G}$ and $V_{F}$ are given by [62]

$$
\begin{aligned}
V_{N}(x, y)=- & \frac{2}{3} \theta(y-x)\left[\frac{5}{3} F(x, y)+\frac{x}{y}+F(x, y) \ln \frac{x}{y}\right]+(x \leftrightarrow \bar{x}, y \leftrightarrow \bar{y}), \\
V_{G}(x, y)= & \left\{\theta(y-x)\left[\frac{67}{9} F(x, y)+\frac{17}{3} \frac{x}{y}+\frac{11}{3} F(x, y) \ln \frac{x}{y}\right]+H(x, y)\right\} \\
& +(x \leftrightarrow \bar{x}, y \leftrightarrow \bar{y}), \\
V_{F}(x, y)=4 & \left\{\theta ( y - x ) \left[-\frac{\pi^{2}}{3} F(x, y)+\frac{x}{y}-\left(\frac{3}{2} F(x, y)-\frac{x}{2 \bar{y}}\right) \ln \frac{x}{y}\right.\right. \\
& \left.-(F(x, y)-F(\bar{x}, \bar{y})) \ln \frac{x}{y} \ln \left(1-\frac{x}{y}\right)+\left(F(x, y)+\frac{x}{2 \bar{y}}\right) \ln ^{2} \frac{x}{y}\right] \\
& \left.-\frac{x}{2 \bar{y}} \ln x(1+\ln x-2 \ln \bar{x})-H(x, y)\right\}+(x \leftrightarrow \bar{x}, y \leftrightarrow \bar{y}),
\end{aligned}
$$


where we have introduced the functions $F(x, y)$ and $H(x, y)$ as follows

$$
\begin{aligned}
F(x, y)= & \frac{x}{y}\left(1+\frac{1}{y-x}\right), \\
H(x, y)= & \theta(x-\bar{y})\left[2(F(x, y)-F(\bar{x}, \bar{y})) \operatorname{Li}_{2}\left(1-\frac{x}{y}\right)-2 F(x, y) \ln x \ln y\right. \\
& \left.+(F(x, y)-F(\bar{x}, \bar{y})) \ln ^{2} y\right]+2 F(x, y) \operatorname{Li}_{2}(\bar{y})[\theta(x-\bar{y})-\theta(y-x)] \\
& +2 \theta(y-x) F(\bar{x}, \bar{y}) \ln y \ln \bar{x}-2 F(x, y) \operatorname{Li}_{2}(x)[\theta(x-\bar{y})-\theta(x-y)] .
\end{aligned}
$$

In order to perform the NLL QCD resummation, it turns out to be convenient to adopt the Gegenbauer expansion of the pion DA

$$
\phi_{\pi}(x, \mu)=6 x \bar{x} \sum_{n=0}^{\infty} a_{n}(\mu) C_{n}^{3 / 2}(2 x-1),
$$

where the LO coefficient $a_{0}(\mu)=1$ is renormalization invariant due to the normalization condition. The exact solution to the two-loop RG equation (2.37) can be constructed from the forward anomalous dimensions and the special conformal anomaly matrix in the Gegenbauer moment space [63, 64], and we obtain (see also [22])

$$
a_{n}(\mu)=E_{V, n}^{\mathrm{NLO}}\left(\mu, \mu_{0}\right) a_{n}\left(\mu_{0}\right)+\frac{\alpha_{s}(\mu)}{4 \pi} \sum_{k=0}^{n-2} E_{V, n}^{\mathrm{LO}}\left(\mu, \mu_{0}\right) d_{V, n}^{k}\left(\mu, \mu_{0}\right) a_{k}\left(\mu_{0}\right),
$$

where both $n$ and $k$ are non-negative even integers and the explicit expressions of $E_{V, n}^{\mathrm{NLO}}$ and $d_{V, n}^{k}$ are collected in appendix A. Inserting (2.48) into (2.33) and employing the technique developed in [65], we obtain

$$
F_{\gamma^{*} \gamma \rightarrow \pi^{0}}^{\mathrm{LP}}\left(Q^{2}\right)=\frac{3 \sqrt{2}\left(Q_{u}^{2}-Q_{d}^{2}\right)}{Q^{2}} f_{\pi} \sum_{n=0}^{\infty} a_{n}(\mu) C_{n}\left(Q^{2}, \mu\right)+\mathcal{O}\left(\alpha_{s}^{2}\right),
$$

where the hard coefficient $C_{n}\left(Q^{2}, \mu\right)$ in the NDR scheme of $\gamma_{5}$ is given by

$$
\begin{aligned}
C_{n}\left(Q^{2}, \mu\right)= & 1+\frac{\alpha_{s}(\mu) C_{F}}{4 \pi}\left\{\left[4 H_{n+1}-\frac{3 n(n+3)+8}{(n+1)(n+2)}\right] \ln \frac{\mu^{2}}{Q^{2}}+4 H_{n+1}^{2}-4 \frac{H_{n+1}+1}{(n+1)(n+2)}\right. \\
& \left.+2\left[\frac{1}{(n+1)^{2}}+\frac{1}{(n+2)^{2}}\right]+3\left[\frac{1}{(n+1)}-\frac{1}{(n+2)}\right]-9\right\},
\end{aligned}
$$

with the harmonic number defined as $H_{n}=\sum_{k=1}^{k=n} 1 / k$.

\section{The subleading-power correction from photon DAs}

In this section we aim at evaluating the power suppressed contribution to the pion-photon form factor due to the hadronic photon effect at $\mathcal{O}\left(\alpha_{s}\right)$ with the LCSR approach. To this end, we construct the following vacuum-to-photon correlation function

$$
\begin{aligned}
G_{\mu}\left(p^{\prime}, q\right) & =\int d^{4} z e^{-i q \cdot z}\left\langle 0\left|\mathrm{~T}\left\{j_{\mu, \perp}^{\mathrm{em}}(z), j_{\pi}(0)\right\}\right| \gamma\left(p^{\prime}\right)\right\rangle \\
& =-g_{\mathrm{em}}^{2} \epsilon_{\mu \nu \alpha \beta}^{\perp} q^{\alpha} p^{\prime \beta} \epsilon_{\nu}\left(p^{\prime}\right) G\left(p^{2}, Q^{2}\right)
\end{aligned}
$$


defined with an electromagnetic current (2.2) carrying a four-momentum $q_{\mu}$ and a pion interpolating current $j_{\pi}$ whose explicit structure is as follows

$$
j_{\pi}=\frac{1}{\sqrt{2}}\left(\bar{u} \gamma_{5} u-\bar{d} \gamma_{5} d\right) .
$$

Here we have introduced the convention $\epsilon_{\mu \nu \alpha \beta}^{\perp} \equiv g_{\mu}^{\rho \perp} \epsilon_{\rho \nu \alpha \beta}$. Following the standard strategy, the primary task for the sum-rule construction consists in the demonstration of QCD factorization for the considered correlation function (3.1) at space-like interpolating momentum $p=p^{\prime}+q$. Due to the appearance of $\gamma_{5}$ in the QCD matrix element (3.1), the nonminimal renormalization of the axial current (dependent on the $\gamma_{5}$ prescription) will be performed to restore the Ward identities of chiral gauge theories order by order in perturbation theory (see the discussion of subsection 3.2 for the details). We will employ both the NDR and HV schemes of the Dirac matrix $\gamma_{5}$ to establish the QCD factorization formula of the transition amplitude (3.1) at $\mathcal{O}\left(\alpha_{s}\right)$ and then derive the NLL resummation improved LCSR for the hadronic photon correction to the $\pi^{0} \gamma^{*} \gamma$ form factor. Furthermore, the power counting rule for the external momenta

$$
|n \cdot p| \sim \bar{n} \cdot p \sim n \cdot p^{\prime} \sim \mathcal{O}\left(\sqrt{Q^{2}}\right),
$$

will be adopted to determine the perturbative matching coefficient entering the factorization formula of $G_{\mu}\left(p^{\prime}, q\right)$ to the one-loop order.

\subsection{The hadronic photon effect at tree level}

QCD factorization for the correlation function (3.1) at tree level can be established by investigating the following four-point QCD amplitude

$$
\widetilde{\Pi}_{\mu}=\int d^{4} z e^{-i q \cdot z}\left\langle 0\left|\mathrm{~T}\left\{j_{\mu, \perp}^{\mathrm{em}}(z), j_{\pi}(0)\right\}\right| q\left(x p^{\prime}\right) \bar{q}\left(\bar{x} p^{\prime}\right)\right\rangle
$$

at LO in $\alpha_{s}$. Evaluating the two diagrams in figure 4 leads to

$$
\begin{aligned}
\widetilde{\Pi}_{\mu}^{(0)} & =-\frac{i g_{\mathrm{em}}}{2 \sqrt{2}} \frac{\bar{n} \cdot p}{Q^{2}}\left[\frac{1}{x r+\bar{x}}+\frac{1}{\bar{x} r+x}\right] \sum_{q=u, d} \eta_{q} Q_{q} \bar{q}\left(\bar{x} p^{\prime}\right) \gamma_{5} \not h \gamma_{\mu, \perp} q\left(x p^{\prime}\right) \\
& =-\frac{i g_{\mathrm{em}}}{2 \sqrt{2}} \frac{\bar{n} \cdot p}{Q^{2}} \sum_{q=u, d} \eta_{q} Q_{q}\left[\frac{1}{x^{\prime} r+\bar{x}^{\prime}}+\frac{1}{\bar{x}^{\prime} r+x^{\prime}}\right] *\left\langle\widetilde{O}_{A, \mu}\left(x, x^{\prime}\right)\right\rangle^{(0)}
\end{aligned}
$$

where $r=-p^{2} / Q^{2}, \eta_{u}=1$ and $\eta_{d}=-1$. The partonic matrix element of the (anti)collinear SCET operator $\widetilde{O}_{A, \mu}$ at tree level is given by

$$
\left\langle\widetilde{O}_{j, \mu}\left(x, x^{\prime}\right)\right\rangle \equiv\left\langle 0\left|\widetilde{O}_{j, \mu}\left(x^{\prime}\right)\right| q\left(x p^{\prime}\right) \bar{q}\left(\bar{x} p^{\prime}\right)\right\rangle=\bar{\chi}\left(\bar{x} p^{\prime}\right) \widetilde{\Gamma}_{j, \mu} \chi\left(x p^{\prime}\right) \delta\left(x-x^{\prime}\right)+\mathcal{O}\left(\alpha_{s}\right) .
$$

The explicit definition of the (anti)-collinear operator $\widetilde{O}_{j, \mu}$ in the momentum space is

$$
\widetilde{O}_{j, \mu}\left(x^{\prime}\right)=\frac{n \cdot p^{\prime}}{2 \pi} \int d \tau e^{i x^{\prime} \tau n \cdot p^{\prime}} \bar{\chi}(0) W_{\bar{c}}(0, \tau n) \widetilde{\Gamma}_{j, \mu} \chi(\tau n),
$$




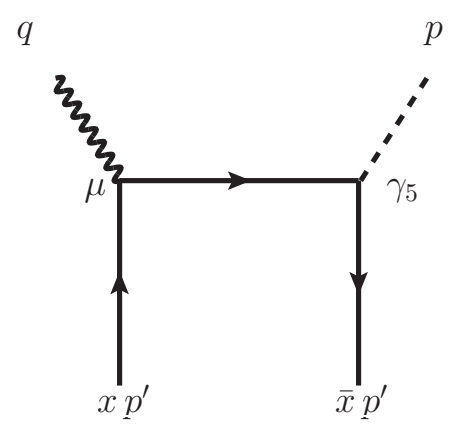

(a)

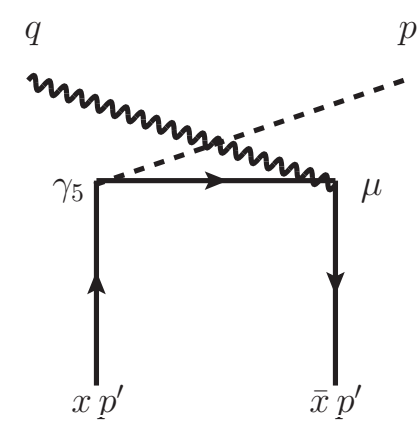

(b)

Figure 4. Diagrammatical representation of the tree-level contribution to the QCD amplitude $\widetilde{\Pi}_{\mu}$ defined in (3.4).

where we have suppressed the flavour indices of $\widetilde{O}_{j, \mu}$ for brevity, $\widetilde{\Gamma}_{A, \mu}=\gamma_{5} \not h \gamma_{\mu, \perp}$ and the corresponding Wilson line is defined as

$$
W_{\bar{c}}(0, \tau n)=\mathrm{P}\left\{\operatorname{Exp}\left[-i g_{s} \int_{0}^{\tau} d \lambda n \cdot A_{\bar{c}}(\lambda n)\right]\right\} .
$$

To achieve the hard-collinear factorization for the correlation function (3.1), we introduce the SCET operator basis $\left\{\widetilde{O}_{1, \mu}, \widetilde{O}_{E, \mu}\right\}$ with

$$
\widetilde{\Gamma}_{1, \mu}=\frac{n^{\alpha}}{2} \epsilon_{\mu \nu \alpha \beta}^{\perp} \sigma^{\nu \beta}, \quad \widetilde{\Gamma}_{E, \mu}=\gamma_{5} \not h \gamma_{\mu, \perp}-\frac{n^{\alpha}}{2} \epsilon_{\mu \nu \alpha \beta}^{\perp} \sigma^{\nu \beta}
$$

where $\widetilde{O}_{E, \mu}$ is an evanescent operator. Applying the operator matching equation including the evanescent operator

$$
\widetilde{\Pi}_{\mu}=-\frac{i g_{\mathrm{em}}}{2 \sqrt{2}} \frac{\bar{n} \cdot p}{Q^{2}} \sum_{q=u, d} \eta_{q} Q_{q} \sum_{i} \widetilde{T}_{i}\left(x^{\prime}\right) *\left\langle\widetilde{O}_{i, \mu}\left(x, x^{\prime}\right)\right\rangle
$$

and expanding all quantities to the tree level, we can readily find that

$$
\widetilde{T}_{1}^{(0)}\left(x^{\prime}\right)=\widetilde{T}_{E}^{(0)}\left(x^{\prime}\right)=\frac{1}{x^{\prime} r+\bar{x}^{\prime}}+\frac{1}{\bar{x}^{\prime} r+x^{\prime}} .
$$

Making use of the leading twist photon DA defined in [35]

$$
\begin{aligned}
& \left\langle 0\left|\bar{\chi}(0) W_{\bar{c}}(0, y) \sigma_{\alpha \beta} \chi(y)\right| \gamma\left(p^{\prime}\right)\right\rangle \\
& =i g_{\mathrm{em}} Q_{q} \chi(\mu)\langle\bar{q} q\rangle(\mu)\left[p_{\beta}^{\prime} \epsilon_{\alpha}\left(p^{\prime}\right)-p_{\alpha}^{\prime} \epsilon_{\beta}\left(p^{\prime}\right)\right] \int_{0}^{1} d u e^{-i u p^{\prime} \cdot y} \phi_{\gamma}(u, \mu),
\end{aligned}
$$

the tree-level factorization formula of the form factor $G\left(p^{2}, Q^{2}\right)$ can be written as

$$
G\left(p^{2}, Q^{2}\right)=-\frac{Q_{u}^{2}-Q_{d}^{2}}{\sqrt{2} Q^{2}} \chi(\mu)\langle\bar{q} q\rangle(\mu) \int_{0}^{1} d x \widetilde{T}_{1}^{(0)}(x) \phi_{\gamma}(x, \mu)+\mathcal{O}\left(\alpha_{s}\right),
$$

where the magnetic susceptibility of the quark condensate $\chi(\mu)$ encodes the dynamical information of the QCD vacuum [66]. 


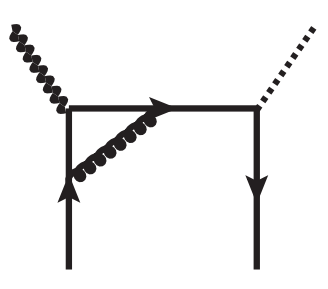

(a)

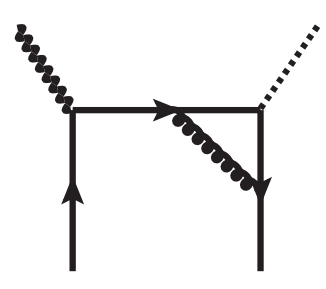

(b)

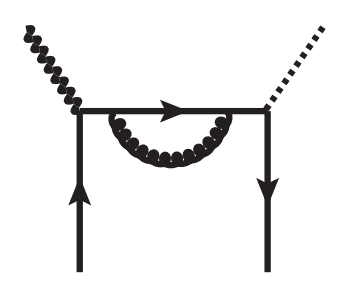

(c)

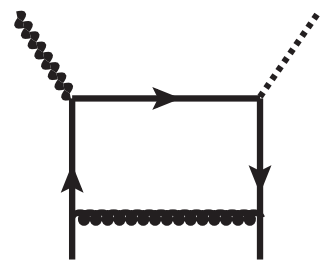

(d)

Figure 5. Diagrammatical representation of the one-loop contribution to the QCD amplitude $\widetilde{\Pi}_{\mu}$ (3.4). The corresponding symmetric diagrams obtained by exchanging the electromagnetic current and the pion interpolating current are not shown.

Applying the standard definition for the pion decay constant

$$
\left\langle 0\left|j_{\pi}\right| \pi(p)\right\rangle=-i f_{\pi} \mu_{\pi}(\mu), \quad \mu_{\pi}(\mu) \equiv \frac{m_{\pi}^{2}}{m_{u}(\mu)+m_{d}(\mu)},
$$

we can write down the hadronic dispersion relation of $G\left(p^{2}, Q^{2}\right)$

$$
G\left(p^{2}, Q^{2}\right)=\frac{f_{\pi} \mu_{\pi}(\mu)}{m_{\pi}^{2}-p^{2}-i 0} F_{\gamma^{*} \gamma \rightarrow \pi^{0}}^{\mathrm{NLP}}\left(Q^{2}\right)+\int_{s_{0}}^{\infty} d s \frac{\rho^{h}\left(s, Q^{2}\right)}{s-p^{2}-i 0}
$$

The final expression of the LCSR for the hadronic photon correction to the pion-photon form factor can then be derived by implementing the continuum subtraction and the Borel transformation with the aid of the parton-hadron duality

$$
\begin{aligned}
F_{\gamma^{*} \gamma \rightarrow \pi^{0}}^{\mathrm{NLP}}\left(Q^{2}\right)= & -\frac{\sqrt{2}\left(Q_{u}^{2}-Q_{d}^{2}\right)}{f_{\pi} \mu_{\pi}(\mu)} \chi(\mu)\langle\bar{q} q\rangle(\mu) \int_{u_{0}}^{1} \frac{d u}{u} \exp \left[-\frac{\bar{u} Q^{2}-u m_{\pi}^{2}}{u M^{2}}\right] \phi_{\gamma}(u, \mu) \\
& +\mathcal{O}\left(\alpha_{s}\right)
\end{aligned}
$$

with $u_{0}=Q^{2} /\left(s_{0}+Q^{2}\right)$. Employing the power counting scheme for the sum rule parameters

$$
s_{0} \sim M^{2} \sim \mathcal{O}\left(\Lambda^{2}\right), \quad \bar{u}_{0} \sim \mathcal{O}\left(\Lambda^{2} / Q^{2}\right),
$$

we can obtain the scaling behaviour of the hadronic photon effect at large $Q^{2}$

$$
\frac{F_{\gamma^{*} \gamma \rightarrow \pi^{0}}^{\mathrm{NLP}}\left(Q^{2}\right)}{F_{\gamma^{*} \gamma \rightarrow \pi^{0}}^{\mathrm{LP}}\left(Q^{2}\right)} \sim \mathcal{O}\left(\frac{\Lambda^{2}}{Q^{2}}\right)
$$

\subsection{The hadronic photon effect at one loop}

To construct the NLL LCSR for the hadronic photon effect, we first need to establish the one-loop factorization formula for the correlation function (3.1) at the leading power in $1 / Q^{2}$. Following the strategy for demonstrating QCD factorization of the leading power contribution presented in section 2 , the perturbative matching coefficient entering the factorization formula of the form factor $G\left(p^{2}, Q^{2}\right)$ can be determined by evaluating the oneloop diagrams for the QCD amplitude $\widetilde{\Pi}_{\mu}(3.4)$ in figure 5 . We will compute the hard 
contributions from these diagrams one-by-one with both the NDR and HV schemes applying the strategy of regions.

The one-loop QCD correction to the electromagnetic vertex diagram displayed in figure $5(\mathrm{a})$ is free of the $\gamma_{5}$ ambiguity and a straightforward calculation yields

$$
\begin{aligned}
\left.\widetilde{\Pi}_{\mu}^{(1 a)}\right|_{\mathrm{NDR}}= & \left.\widetilde{\Pi}_{\mu}^{(1 a)}\right|_{\mathrm{HV}}=\frac{i g_{\mathrm{em}}}{2 \sqrt{2}} \frac{\bar{n} \cdot p}{Q^{2}} \frac{\alpha_{s} C_{F}}{4 \pi} \sum_{q=u, d} \eta_{q} Q_{q}\left\langle\widetilde{O}_{1, \mu}\left(x, x^{\prime}\right)\right\rangle^{(0)} *\left\{\frac{1}{x^{\prime} r+\bar{x}^{\prime}} \frac{1}{x^{\prime} \bar{r}}\right. \\
& \times\left(\left[2 \ln \left(x^{\prime} r+\bar{x}^{\prime}\right)+x^{\prime} \bar{r}\right]\left[\frac{1}{\epsilon}+\ln \frac{\mu^{2}}{Q^{2}}-\frac{1}{2} \ln \left(x^{\prime} r+\bar{x}^{\prime}\right)-\frac{x^{\prime} \bar{r}}{4}+\frac{3}{2}\right]\right. \\
& \left.\left.+\frac{x^{\prime} \bar{r}\left(x^{\prime} \bar{r}+10\right)}{4}\right)\right\}+\ldots
\end{aligned}
$$

where the term proportional to $\left\langle\widetilde{O}_{E, \mu}\left(x, x^{\prime}\right)\right\rangle$ is not shown explicitly. Due to the appearance of $\gamma_{5}$ in the pion interpolating current, the one-loop QCD correction to the pion vertex diagram depends on the $\gamma_{5}$ prescription employed in the reduction of the Dirac algebra. Computing the hard contribution from the one-loop diagram 5(b) in both the NDR and HV schemes gives

$$
\begin{aligned}
\left.\widetilde{\Pi}_{\mu}^{(1 b)}\right|_{\mathrm{NDR}}= & -\frac{i g_{\mathrm{em}}}{2 \sqrt{2}} \frac{\bar{n} \cdot p}{Q^{2}} \frac{\alpha_{s} C_{F}}{4 \pi} \sum_{q=u, d} \eta_{q} Q_{q}\left\langle\widetilde{O}_{1, \mu}\left(x, x^{\prime}\right)\right\rangle^{(0)} *\left\{\frac{1}{x^{\prime} r+\bar{x}^{\prime}}\right. \\
& \times\left(2\left[\frac{r}{\bar{x}^{\prime} \bar{r}} \ln \left(\frac{x^{\prime} r+\bar{x}^{\prime}}{r}\right)+1\right]\left[\frac{1}{\epsilon}+\ln \frac{\mu^{2}}{Q^{2}}-\frac{1}{2} \ln r-\frac{1}{2} \ln \left(x^{\prime} r+\bar{x}^{\prime}\right)-\frac{\bar{x}^{\prime} \bar{r}}{2 r}\right]\right. \\
& \left.\left.+\frac{\bar{x}^{\prime} \bar{r}}{r}+2\right)\right\}+\ldots, \\
\left.\widetilde{\Pi}_{\mu}^{(1 b)}\right|_{\mathrm{HV}}= & \left.\widetilde{\Pi}_{\mu}^{(1 b)}\right|_{\mathrm{NDR}}+\frac{2 \alpha_{s} C_{F}}{\pi} \widetilde{\Pi}_{\mu}^{(0 a)}+\ldots,
\end{aligned}
$$

where $\widetilde{\Pi}_{\mu}^{(0 a)}$ represents the tree-level contribution to the diagram 4(a) and can be obtained from (3.5) by keeping only the first term in the square bracket. The self-energy correction to the intermediate hard propagator displayed in figure $5(\mathrm{c})$ is independent of the $\gamma_{5}$ prescription in the $D$-dimensional space and we obtain

$$
\begin{aligned}
\left.\widetilde{\Pi}_{\mu}^{(1 c)}\right|_{\mathrm{NDR}}= & \left.\widetilde{\Pi}_{\mu}^{(1 c)}\right|_{\mathrm{HV}}=\frac{i g_{\mathrm{em}}}{2 \sqrt{2}} \frac{\bar{n} \cdot p}{Q^{2}} \frac{\alpha_{s} C_{F}}{4 \pi} \sum_{q=u, d} \eta_{q} Q_{q}\left\langle\widetilde{O}_{1, \mu}\left(x, x^{\prime}\right)\right\rangle^{(0)} \\
& *\left\{\frac{1}{x^{\prime} r+\bar{x}^{\prime}}\left[\frac{1}{\epsilon}+\ln \frac{\mu^{2}}{Q^{2}}-\ln \left(x^{\prime} r+\bar{x}^{\prime}\right)+1\right]\right\}+\ldots
\end{aligned}
$$

We finally turn to compute the hard contribution from the one-loop box diagram shown in figure $5(\mathrm{~d})$, which depends on the actual prescription of $\gamma_{5}$ adopted in the calculation of the corresponding QCD amplitude. Evaluating the contribution from the box diagram with both the NDR and HV schemes, we find that the corresponding hard coefficients only contribute at $\mathcal{O}(\epsilon)$, vanishing in four-dimensional space. Explicitly,

$$
\left.\widetilde{\Pi}_{\mu}^{(1 d)}\right|_{\mathrm{NDR}}=\left.\widetilde{\Pi}_{\mu}^{(1 d)}\right|_{\mathrm{HV}}=0 .
$$


Collecting different pieces together, we derive the NLO QCD correction to the four-point amplitude $\widetilde{\Pi}_{\mu}$ as follows

$$
\widetilde{\Pi}_{\mu}^{(1)}=-\frac{i g_{\mathrm{em}}}{2 \sqrt{2}} \frac{\bar{n} \cdot p}{Q^{2}} \sum_{q=u, d} \eta_{q} Q_{q}\left\langle\widetilde{O}_{1, \mu}\left(x, x^{\prime}\right)\right\rangle^{(0)} * \widetilde{A}_{1, \mathrm{hard}}\left(x^{\prime}\right)+\ldots
$$

where the renormalization prescription dependent hard amplitude $\widetilde{A}_{1 \text {,hard }}$ is given by

$$
\begin{aligned}
\left.\widetilde{A}_{1, \mathrm{hard}}\left(x^{\prime}\right)\right|_{\mathrm{NDR}}= & \frac{\alpha_{s} C_{F}}{4 \pi}\left\{\frac { 1 } { x ^ { \prime } r + \overline { x } ^ { \prime } } \left[\frac { 2 } { x ^ { \prime } \overline { x } ^ { \prime } \overline { r } } \left(\left(\left(x^{\prime} r-\bar{x}^{\prime}\right) \ln \left(x^{\prime} r+\bar{x}^{\prime}\right)-x^{\prime} r \ln r\right)\right.\right.\right. \\
& \left.\left(\frac{1}{\epsilon}+\ln \frac{\mu^{2}}{Q^{2}}-\frac{1}{2} \ln \left(x^{\prime} r+\bar{x}^{\prime}\right)-\frac{1}{2} \ln r\right)\right) \\
& \left.\left.-\frac{1}{x^{\prime} \bar{r}}(\ln r+3) \ln \left(x^{\prime} r+\bar{x}^{\prime}\right)-3\right]+\left(x^{\prime} \leftrightarrow \bar{x}^{\prime}\right)\right\} \\
\left.\widetilde{A}_{1, \text { hard }}\left(x^{\prime}\right)\right|_{\mathrm{HV}}= & \left.\widetilde{A}_{1, \text { hard }}\left(x^{\prime}\right)\right|_{\mathrm{NDR}}+\frac{2 \alpha_{s} C_{F}}{\pi} \widetilde{T}_{1}^{(0)}\left(x^{\prime}\right) .
\end{aligned}
$$

Applying the strategy to implement the IR subtraction for the four-point QCD amplitude $\Pi_{\mu}$ discussed in section 2, the master formula for the one-loop hard coefficient of the physical SCET operator $\widetilde{O}_{1, \mu}$ can be written as

$$
\widetilde{T}_{1}^{(1)}=\widetilde{A}_{1}^{(1)}-\widetilde{T}_{1}^{(0)} * \widetilde{Z}_{11}^{(1)}+\widetilde{T}_{E}^{(0)} * \widetilde{M}_{E 1}^{(1) \text { off }}=\widetilde{A}_{1, \text { hard }}^{(1)}+\widetilde{T}_{E}^{(0)} * \widetilde{M}_{E 1}^{(1) \text { off }}
$$

where the bare matrix element $\widetilde{M}_{E 1}^{(1) \text { off }}$ represents the QCD mixing of the evanescent operator $\widetilde{O}_{E, \mu}$ into $\widetilde{O}_{1, \mu}$ at one loop. It is obvious that the infrared subtraction term $\widetilde{T}_{E}^{(0)} * \widetilde{M}_{E 1}^{(1) \text { off }}$ suffers from the $\gamma_{5}$ ambiguity in dimensional regularization. The corresponding SCET diagrams at one loop are in analogy to that displayed in figure 3 , but with the vertex " $\otimes$ " indicating an insertion of $\widetilde{O}_{E, \mu}$. Computing these effective diagrams with dimensional regularization applied to the UV divergences and with the IR singularities regularized by the fictitious gluon mass, we find that $\widetilde{M}_{E 1}^{(1) \text { off }}$ vanishes at one loop with the NDR scheme of $\gamma_{5}$ and it receives a nonvanishing contribution of $\mathcal{O}(\epsilon)$ with the $\mathrm{HV}$ scheme of $\gamma_{5}$ from the effective diagram with a collinear gluon exchange between two external quarks. We are then led to conclude that

$$
\left.\widetilde{T}_{E}^{(0)} * \widetilde{M}_{E 1}^{(1) \mathrm{off}}\right|_{\mathrm{NDR}}=\left.\widetilde{T}_{E}^{(0)} * \widetilde{M}_{E 1}^{(1) \mathrm{off}}\right|_{\mathrm{HV}}=0
$$

Inserting (3.28) into (3.27) yields

$$
\widetilde{T}_{1}^{(1)}=\widetilde{A}_{1, \text { hard }}^{(1)}
$$

for both the NDR and HV schemes of the $\gamma_{5}$ matrix, with $\widetilde{A}_{1, \text { hard }}^{(1)}$ presented in (3.25) and (3.26). We mention in passing that the $\gamma_{5}$ scheme dependence of the short-distance function $\widetilde{T}_{1}^{(1)}$ will not be cancelled by the one-loop QCD correction to the twist-2 photon DA defined by the light-cone matrix element of the tensor current, which is clearly free of the $\gamma_{5}$ ambiguity in dimensional regularization, and the $\gamma_{5}$ ambiguity of $\widetilde{A}_{1 \text {,hard }}^{(1)}$ can be traced back to the renormalization prescription dependence of the QCD amplitude (3.4) itself. 
To preserve the one-loop character of the axial anomaly, an additional finite counterterm must be introduced [11]

$$
Z_{\mathrm{HV}}^{P}(\mu)=1-\frac{2 \alpha_{s}(\mu) C_{F}}{\pi}+\mathcal{O}\left(\alpha_{s}^{2}\right)
$$

when performing the UV renormalization of the pseudoscalar current in the HV scheme. Making use of (3.11), (3.25), (3.26) and (3.29), it is then straightforward to verify that

$$
Z_{\mathrm{HV}}^{P}(\mu)\left[\widetilde{T}_{1}^{(0)}\left(x^{\prime}\right)+\widetilde{T}_{1}^{(1)}\left(x^{\prime}, \mu\right)\right]_{\mathrm{HV}}=\left[\widetilde{T}_{1}^{(0)}\left(x^{\prime}\right)+\widetilde{T}_{1}^{(1)}\left(x^{\prime}, \mu\right)\right]_{\mathrm{NDR}}
$$

at one loop, which provides a nontrivial check to justify the obtained one-loop hard amplitude $\widetilde{T}_{1}$. The NLO factorization formula for the vacuum-to-photon correlation function can be further derived as follows

$$
G\left(p^{2}, Q^{2}\right)=-\frac{Q_{u}^{2}-Q_{d}^{2}}{\sqrt{2} Q^{2}} \chi(\mu)\langle\bar{q} q\rangle(\mu) \int_{0}^{1} d x\left[\widetilde{T}_{1}^{(0)}(x)+\widetilde{T}_{1}^{(1)}(x, \mu)\right]_{\mathrm{NDR}} \phi_{\gamma}(x, \mu)+\mathcal{O}\left(\alpha_{s}^{2}\right) .
$$

With the NLO hard coefficient function $\widetilde{T}_{1}^{(1)}$ at hand, we can also obtain the one-loop short-distance function entering the factorization formula of the $H \rightarrow J / \psi \gamma$ form factor at leading power in $1 / m_{H}^{2}$ by taking the $r \rightarrow \infty$ limit of $\widetilde{T}_{1}^{(1)}$ and by performing the analytical continuation in the variable $p^{2}$, which reproduces the expression displayed in (3.17) of [67] (see also $[68,69]$ ) computed from an alternative approach precisely.

We are now in a position to demonstrate the factorization-scale independence of (3.32) by employing the RG equation of the leading twist photon DA

$$
\mu^{2} \frac{d}{d \mu^{2}}\left[\chi(\mu)\langle\bar{q} q\rangle(\mu) \phi_{\gamma}(x, \mu)\right]=\int_{0}^{1} d y \widetilde{V}(x, y)\left[\chi(\mu)\langle\bar{q} q\rangle(\mu) \phi_{\gamma}(y, \mu)\right]
$$

with the perturbative expansion of the evolution kernel

$$
\widetilde{V}(x, y)=\sum_{n=0}\left(\frac{\alpha_{s}}{4 \pi}\right)^{n+1} \widetilde{V}_{n}(x, y) .
$$

The one-loop renormalization kernel $\widetilde{V}_{0}(x, y)$ is given by [70]

$$
\widetilde{V}_{0}(x, y)=2 C_{F}\left[\frac{\bar{x}}{\bar{y}} \frac{1}{x-y} \theta(x-y)+\frac{x}{y} \frac{1}{y-x} \theta(y-x)\right]_{+}-C_{F} \delta(x-y) .
$$

Taking into account the factorization scale dependence of $\widetilde{T}_{1}^{(1)}(x, \mu)$, we can further deduce $\frac{d}{d \ln \mu} G\left(p^{2}, Q^{2}\right)=-\frac{3}{2} \frac{\alpha_{s}(\mu) C_{F}}{\pi} \frac{Q_{u}^{2}-Q_{d}^{2}}{\sqrt{2} Q^{2}} \chi(\mu)\langle\bar{q} q\rangle(\mu) \int_{0}^{1} d x \widetilde{T}_{1}^{(0)}(x) \phi_{\gamma}(x, \mu)+\mathcal{O}\left(\alpha_{s}^{2}\right)$.

The residual $\mu$-dependence of $G\left(p^{2}, Q^{2}\right)$ originates from the UV renormalization of the QCD pseudoscalar current defining the correlation function (3.1). Taking advantage of the evolution equation of the QCD renormalization constant for the pseudoscalar current [71, 72]

$$
\frac{d}{d \ln \mu} \ln Z_{P}(\mu)=\sum_{n=0}\left(\frac{\alpha_{s}(\mu)}{4 \pi}\right)^{n+1} \gamma_{P}^{(n)}, \quad \gamma_{P}^{(0)}=6 C_{F},
$$


and distinguishing the renormalization scale of the QCD current from the factorization scale due to the IR subtraction (see [73] for more details), we can then find that the expression for the form factor $G\left(p^{2}, Q^{2}\right)$ (3.32) is indeed factorization-scale invariant at $\mathcal{O}\left(\alpha_{s}\right)$.

We proceed to perform the NLL resummation for the parametrically large logarithms in the short-distance function $\widetilde{T}_{1}^{(1)}$, which can be achieved alternatively by fixing the factorization scale as $\mu \sim \sqrt{Q^{2}}$ and by evolving the twist-2 photon DA from the hadronic scale to that scale. To this end, we need the two-loop coefficient of the evolution kernel $\widetilde{V}(x, y)[61,74,75]$

$$
\widetilde{V}_{1}(x, y)=\frac{N_{f}}{2} C_{F} \widetilde{V}_{N}(x, y)+C_{F} C_{A} \widetilde{V}_{G}(x, y)+C_{F}^{2} \widetilde{V}_{F}(x, y),
$$

where the explicit expressions of the kernel functions are [74]

$$
\begin{aligned}
\widetilde{V}_{N}(x, y)= & \left\{-\frac{4}{3}\left[2 \theta(y-x) \widetilde{F}(x, y)\left(\ln \frac{x}{y}+\frac{5}{3}\right)\right]_{+}+(x \leftrightarrow \bar{x}, y \leftrightarrow \bar{y})\right\}+\frac{26}{9} \delta(x-y), \\
\widetilde{V}_{G}(x, y)= & \left\{-2\left[\theta(y-x) \frac{x}{y}+\theta(y-\bar{x}) \frac{\bar{x}}{y}\right]+(x \leftrightarrow \bar{x}, y \leftrightarrow \bar{y})\right\}-\widetilde{H}(x, y) \\
& +\left\{\left[2 \theta(y-x) \widetilde{F}(\bar{x}, \bar{y})\left(\frac{11}{3} \ln \frac{x}{y}+\frac{67}{9}-\frac{\pi^{2}}{3}\right)\right]+(x \leftrightarrow \bar{x}, y \leftrightarrow \bar{y})\right\} \\
& +\left[-\frac{221}{18}-12 \zeta(3)+\frac{4 \pi^{2}}{3}\right] \delta(x-y), \\
\widetilde{V}_{F}(x, y)= & \left\{4\left[\theta(y-x) \frac{x}{y}+\theta(y-\bar{x}) \frac{\bar{x}}{y}\right]+(x \leftrightarrow \bar{x}, y \leftrightarrow \bar{y})\right\}+2 \widetilde{H}(x, y) \\
& +\left\{4 \left[\theta ( y - x ) \left(\widetilde{F}(x, y) \ln \frac{x}{y}+\frac{1}{y \bar{y}} \ln x \ln \bar{x}-\frac{3}{2} \widetilde{F}(x, y) \ln \frac{x}{y}\right.\right.\right. \\
& \left.\left.\left.-(\widetilde{F}(x, y)-\widetilde{F}(\bar{x}, \bar{y})) \ln \frac{x}{y} \ln \left(1-\frac{x}{y}\right)\right)\right]+(x \leftrightarrow \bar{x}, y \leftrightarrow \bar{y})\right\} \\
& +4\left[\frac{11}{8}+6 \zeta(3)-\frac{2 \pi^{2}}{3}\right] \delta(x-y),
\end{aligned}
$$

with

$$
\begin{aligned}
\widetilde{F}(x, y)= & \frac{x}{y} \frac{1}{y-x}, \\
\widetilde{H}(x, y)= & -4\left[\theta(y-x)\left(\widetilde{F}(\bar{x}, \bar{y}) \ln \bar{x} \ln y-\widetilde{F}(x, y)\left[\operatorname{Li}_{2}(x)+\operatorname{Li}_{2}(\bar{y})\right]+\frac{\pi^{2}}{6} \widetilde{F}(x, y)\right)\right. \\
& +\theta(x-\bar{y})\left(\left[\operatorname{Li}_{2}\left(1-\frac{x}{y}\right)+\frac{1}{2} \ln ^{2} x\right]+\widetilde{F}(x, y)\left[\operatorname{Li}_{2}(\bar{y})-\ln x \ln y\right]\right. \\
& \left.\left.+\widetilde{F}(\bar{x}, \bar{y}) \operatorname{Li}_{2}(\bar{x})\right)\right]+(x \leftrightarrow \bar{x}, y \leftrightarrow \bar{y}) .
\end{aligned}
$$

Applying the Gegenbauer expansion of the twist-2 photon DA [35]

$$
\phi_{\gamma}(x, \mu)=6 x \bar{x} \sum_{n=0}^{\infty} b_{n}(\mu) C_{n}^{3 / 2}(2 x-1),
$$


and implementing the conformal consistency relation discussed in [63], the two-loop evolution of the Gegenbauer moment $b_{n}\left(\mu_{0}\right)$ can be constructed as follows

$$
\begin{aligned}
\chi(\mu)\langle\bar{q} q\rangle(\mu) b_{n}(\mu)= & E_{T, n}^{\mathrm{NLO}}\left(\mu, \mu_{0}\right) \chi\left(\mu_{0}\right)\langle\bar{q} q\rangle\left(\mu_{0}\right) b_{n}\left(\mu_{0}\right) \\
& +\frac{\alpha_{s}(\mu)}{4 \pi} \sum_{k=0}^{n-2} E_{T, n}^{\mathrm{LO}}\left(\mu, \mu_{0}\right) d_{T, n}^{k}\left(\mu, \mu_{0}\right) \chi\left(\mu_{0}\right)\langle\bar{q} q\rangle\left(\mu_{0}\right) b_{n}\left(\mu_{0}\right),
\end{aligned}
$$

with even $k, n \geq 0$. The detailed expressions the evolution functions $E_{T, n}^{\mathrm{NLO}}$ and $d_{T, n}^{k}$ can be found in appendix A. Combining everything together we arrive at the NLL resummation improved factorization formula

$$
G\left(p^{2}, Q^{2}\right)=-\frac{\left(Q_{u}^{2}-Q_{d}^{2}\right)}{\sqrt{2} Q^{2}} \sum_{n=0}\left[\chi(\mu)\langle\bar{q} q\rangle(\mu) b_{n}(\mu)\right] \widetilde{C}_{n}\left(Q^{2}, \mu\right)+\mathcal{O}\left(\alpha_{s}^{2}\right) .
$$

where the perturbative matching coefficient $\widetilde{C}_{n}\left(Q^{2}, \mu\right)$ is defined by

$$
\widetilde{C}_{n}\left(Q^{2}, \mu\right)=\int_{0}^{1} d x\left[\widetilde{T}_{1}^{(0)}(x)+\widetilde{T}_{1}^{(1)}(x, \mu)\right]_{\mathrm{NDR}}\left[6 x \bar{x} C_{n}^{3 / 2}(2 x-1)\right] .
$$

We will not present the analytical result of $\widetilde{C}_{n}\left(Q^{2}, \mu\right)$ by evaluating the appeared convolution integral explicitly, since the continuum subtraction needs to be performed for the dispersion representation of (3.47) in order to construct the desired LCSRs for the hadronic photon correction to the pion-photon form factor.

Employing the spectral representations of the convolution integrals displayed in appendix B, it is straightforward to derive the dispersion form of the NLL factorization formula

$$
\begin{aligned}
G\left(p^{2}, Q^{2}\right)= & -\frac{\sqrt{2}\left(Q_{u}^{2}-Q_{d}^{2}\right)}{Q^{2}} \chi(\mu)\langle\bar{q} q\rangle(\mu) \int_{0}^{\infty} \frac{d s}{s-p^{2}-i 0} \\
& \times\left[\rho^{(0)}\left(s, Q^{2}\right)+\frac{\alpha_{s} C_{F}}{4 \pi} \rho^{(1)}\left(s, Q^{2}\right)\right]
\end{aligned}
$$

where we have exploited the symmetric property of the photon DA $\phi_{\gamma}(x, \mu)=\phi_{\gamma}(\bar{x}, \mu)$ due to the charge-parity conservation. The resulting QCD spectral densities $\rho^{(i)}\left(s, Q^{2}\right)$ $(i=0,1)$ can be written as

$$
\begin{aligned}
\rho^{(0)}\left(s, Q^{2}\right)= & \frac{Q^{2}}{Q^{2}+s} \phi_{\gamma}\left(\frac{Q^{2}}{Q^{2}+s}, \mu\right) \\
\rho^{(1)}\left(s, Q^{2}\right)= & 2 \int_{0}^{1} \frac{d u}{\bar{u}}\left\{\theta\left(u-\frac{Q^{2}}{Q^{2}+s}\right) \frac{Q^{2}}{Q^{2}+s}\left[\frac{\bar{u}-u}{u} \ln \left(\frac{\mu^{2}}{u s-\bar{u} Q^{2}}\right)+\frac{3}{2} \frac{\bar{u}}{u}\right]\right. \\
& \left.+\ln \left(\frac{\mu^{2}}{s}\right)\left[\frac{Q^{2}}{Q^{2}+s}-\mathcal{P} \frac{\bar{u} Q^{2}}{\bar{u} Q^{2}-u s}\right]\right\} \phi_{\gamma}(u, \mu) \\
& +\frac{Q^{2}}{Q^{2}+s} \int_{0}^{1} d u \theta\left(u-\frac{Q^{2}}{Q^{2}+s}\right)\left\{2 \operatorname { l n } ( \frac { u s - \overline { u } Q ^ { 2 } } { Q ^ { 2 } } ) \left[\ln \left(\frac{\mu^{2}}{u s-\bar{u} Q^{2}}\right)\right.\right. \\
& \left.\left.+\ln \left(\frac{\mu^{2}}{Q^{2}}\right)+\frac{3}{2}\right]-\ln ^{2}\left(\frac{\mu^{2}}{Q^{2}}\right)+\ln ^{2}\left(\frac{\mu^{2}}{s}\right)-\frac{\pi^{2}}{3}+3\right\} \frac{d}{d u} \phi_{\gamma}(u, \mu),
\end{aligned}
$$


where $\mathcal{P}$ indicates the principle-value prescription. The NLL LCSRs for the subleading power contribution to the $\pi^{0} \gamma^{*} \gamma$ form factor can be further derived as

$$
\begin{aligned}
F_{\gamma^{*} \gamma \rightarrow \pi^{0}}^{\mathrm{NLP}}\left(Q^{2}\right)= & -\frac{\sqrt{2}\left(Q_{u}^{2}-Q_{d}^{2}\right)}{f_{\pi} \mu_{\pi}(\mu) Q^{2}} \chi(\mu)\langle\bar{q} q\rangle(\mu) \int_{0}^{s_{0}} d s \exp \left[-\frac{s-m_{\pi}^{2}}{M^{2}}\right] \\
& \times\left[\rho^{(0)}\left(s, Q^{2}\right)+\frac{\alpha_{s} C_{F}}{4 \pi} \rho^{(1)}\left(s, Q^{2}\right)\right]+\mathcal{O}\left(\alpha_{s}^{2}\right) .
\end{aligned}
$$

Collecting different contributions together, we now present the final expression for the pion-photon form factor including the twist-4 correction computed in [22, 26]

$$
F_{\gamma^{*} \gamma \rightarrow \pi^{0}}\left(Q^{2}\right)=F_{\gamma^{*} \gamma \rightarrow \pi^{0}}^{\mathrm{LP}}\left(Q^{2}\right)+F_{\gamma^{*} \gamma \rightarrow \pi^{0}}^{\mathrm{NLP}}\left(Q^{2}\right)+F_{\gamma^{*} \gamma \rightarrow \pi^{0}}^{\mathrm{tw}-4}\left(Q^{2}\right)
$$

where the explicit expressions of $F_{\gamma^{*} \gamma \rightarrow \pi^{0}}^{\mathrm{LP}}$ and $F_{\gamma^{*} \gamma \rightarrow \pi^{0}}^{\mathrm{NLP}}$ are displayed in (2.50) and (3.51), respectively. The obtained factorization formula of $F_{\gamma^{*} \gamma \rightarrow \pi^{0}}^{\mathrm{tw}-4}$ from both the two-particle and the three-particle pion DAs at tree level reads

$$
F_{\gamma^{*} \gamma \rightarrow \pi^{0}}^{\mathrm{tw}-4}\left(Q^{2}\right)=-\frac{\sqrt{2}\left(Q_{u}^{2}-Q_{d}^{2}\right)}{Q^{4}} \int_{0}^{1} d x \frac{\mathbb{F}_{\pi}(x, \mu)}{x^{2}}+\mathcal{O}\left(\alpha_{s}\right)
$$

where the definition of the twist-4 pion DA $\mathbb{F}_{\pi}$ can be found in (38) of [22] and keeping only the leading conformal spin (i.e., "S"-wave) contribution we obtain

$$
\mathbb{F}_{\pi}(x, \mu)=\frac{80}{3} \delta_{\pi}^{2}(\mu) x^{2}(1-x)^{2} .
$$

The nonperturbative parameter $\delta_{\pi}^{2}$ is defined by the local QCD matrix element

$$
\left\langle 0\left|g_{s} \bar{q} \tilde{G}_{\mu \nu} \gamma^{\nu} q\right| \pi(p)\right\rangle=i f_{\pi} \delta_{\pi}^{2}(\mu) p_{\mu}
$$

with the renormalization-scale evolution at one loop

$$
\delta_{\pi}^{2}(\mu)=\left[\frac{\alpha_{s}(\mu)}{\alpha_{s}\left(\mu_{0}\right)}\right]^{\frac{32}{9 \beta_{0}}} \delta_{\pi}^{2}\left(\mu_{0}\right) .
$$

Several comments on the general structure of the $\pi^{0} \gamma^{*} \gamma$ form factor (3.52) are in order.

- It is apparent that the twist-four correction to the $\pi^{0} \gamma^{*} \gamma$ form factor is suppressed by a factor of $\delta_{\pi}^{2} / Q^{2}$ compared with that of the leading twist contribution. Such subleading power contribution turns out to be numerically significant at $Q^{2} \leq 5 \mathrm{GeV}^{2}$ due to the large prefactor "80/3" entering the asymptotic expression of $\mathbb{F}_{\pi}(x, \mu)$, however, it is still far from sufficient to generate the scaling violation at $Q^{2} \sim 40 \mathrm{GeV}^{2}$ indicated by the BaBar measurement [21]. Furthermore, it is of high interest to compute the NLO correction to the twist-four contribution in order to develop a better understanding of factorization properties of the high twist effects, where the infrared subtraction for constructing the factorization formula is complicated by the mixing of different twist-four pion DAs under the QCD renormalization. 
- The twist-six correction to the pion-photon form factor computed from the dispersion approach [22] is partially absorbed into the hadronic photon effect $F_{\gamma^{*} \gamma \rightarrow \pi^{0}}^{\mathrm{NLP}}\left(Q^{2}\right)$ displayed in (3.51). The precise correspondence of distinct contributions in two frameworks cannot be established without identifying the operator-level definitions of the "soft" corrections in [22], which originate from the nonperturbative modification of the QCD spectral density appeared in the dispersion form of the $\pi^{0} \gamma^{*} \gamma^{*}$ form factor.

- The subleading power corrections from the yet higher twist pion/photon DAs, which are not taken into account in this work, are conjectured to be suppressed by only one power of $\Lambda^{2} / Q^{2}$ due to the absent correspondence between the twist counting and the large-momentum expansion [22]. A direct calculation of the two-particle and three-particle corrections to the pion-photon form factor from the twist-three and twist-four photon DAs based upon the LCSR approach is in demand to verify this interesting hypothesis.

- We do not include the NNLO QCD correction to the leading power contribution in the large $\beta_{0}$ approximation [8] on account of the absence of a complete NNLO contribution, which also necessitates the three-loop evolution equation of the twisttwo pion DA [76] to obtain the factorization formula at the next-to-next-to-leadinglogarithmic accuracy. A recent discussion of the NNLO radiative corrections in the framework of the dispersion approach can be found in [30].

\section{Numerical analysis}

We are now ready to explore the phenomenological consequences of the hadronic photon correction to the pion-photon form factor applying the master formula (3.52). In doing so, we will first need to specify the non-perturbative models for the twist-2 pion DA, the magnetic susceptibility $\chi(\mu)$, the Gegenbauer moments of the photon DA, and to determine the "internal" sum rule parameters entering the expression (3.51).

\subsection{Theory input parameters}

The fundamental ingredients entering the NLL factorization formula of the leading power contribution are the Gegenbauer moments of the twist-2 pion DA. Tremendous efforts have been devoted to the determinations of the lowest moment $a_{2}(\mu)$ from the direct calculations with the QCD sum rules pioneered by Chernyak and Zhitnitsky (CZ) [77] and with the lattice simulations, and from the indirect calculations by matching the LCSR predictions with the experimental data. To quantify the systematic uncertainty from the Gegenbauer moments, we will consider the following four models for the leading twist pion DA

$$
\begin{aligned}
a_{2}(1.0 \mathrm{GeV}) & =0.21_{-0.06}^{+0.07}, \quad a_{4}(1.0 \mathrm{GeV})=-\left(0.15_{-0.09}^{+0.10}\right), & & (\mathrm{BMS}) ; \\
a_{2}(1.0 \mathrm{GeV}) & =0.17 \pm 0.08, \quad a_{4}(1.0 \mathrm{GeV})=0.06 \pm 0.10, & & (\text { KMOW }) ; \\
a_{n}(1.0 \mathrm{GeV}) & =\frac{2 n+3}{3 \pi}\left(\frac{\Gamma[(n+1) / 2]}{\Gamma[(n+4) / 2]}\right)^{2}, & & (\text { Hol. }) ; \\
a_{2}(1.0 \mathrm{GeV}) & =0.5, \quad a_{n>2}(1.0 \mathrm{GeV})=0, & & (\mathrm{CZ}) .
\end{aligned}
$$


The obtained Gegenbauer coefficients in the Bakulev-Mikhailov-Stefanis (BMS) model $[30,78]$ are computed from the QCD sum rules with non-local condensates absorbing the high-order terms in the operator-product-expansion (OPE) partially (see, however, [79]). The first and second nontrivial Gegenbauer moments of the KMOW model [80] are determined by comparing the LCSR predictions for the pion electromagnetic form factor, including the NLO correction to the twist-2 effect and the subleading terms up to twist-6, with the intermediate- $Q^{2}$ data from the JLab experiment. The holographic model of the twist-2 pion DA [81]

$$
\phi_{\pi}^{\mathrm{Hol}}\left(x, \mu_{0}\right)=\frac{8}{\pi} \sqrt{x(1-x)}
$$

is motivated by the correspondence between the string theory in the five-dimensional antide Sitter space and conformal field theories in the physical space-time (see also [82] for a similar end-point behaviour of the pion DA) and implementing the Gegenbauer expansion of $\phi_{\pi}^{\mathrm{Hol}}\left(x, \mu_{0}\right)$ leads to the expression of $a_{n}$ displayed in (4.1). For the phenomenological analysis of the $\pi^{0} \gamma^{*} \gamma$ form factor, we will truncate the expansion of the "holographic" model at $n=12$, which was demonstrated to be a good approximation in [22]. It needs to be pointed our that the values of the second Gegenbauer coefficient in the first three models of (4.1) are in line with the recent lattice determinations [83] within the theory uncertainties and the $\mathrm{CZ}$ model is introduced for the illustration purpose to understand the model dependence of the predictions for the pion-photon form factor.

The normalization parameter for the twist-four pion DAs will be taken as $\delta_{\pi}^{2}(1 \mathrm{GeV})=$ $(0.2 \pm 0.04) \mathrm{GeV}^{2}$ computed from the QCD sum rules [84] (see also [85]). We further adopt the value of the quark condensate density $\langle\bar{q} q\rangle(1 \mathrm{GeV})=-\left(256_{-16}^{+14} \mathrm{MeV}\right)^{3}$ determined in [80]. A key nonperturbative quantity appearing in the twist-2 photon DA is the magnetic susceptibility of the quark condensate $\chi(\mu)$ describing a response of the QCD vacuum in the presence of an external photon field. Different QCD-based approaches have been proposed to evaluate $\chi(\mu)$ (see, e.g., [35, 86, 87]) with the aid of the resonance information from the experimental data and the interval $\chi(1 \mathrm{GeV})=(3.15 \pm 0.3) \mathrm{GeV}^{-2}$ [35] will be employed in the numerical calculations. In contrast, our understanding of the higher Gegenbauer moments of the leading twist photon DA is rather limited, even for the leading non-asymptotic correction due to $b_{2}\left(\mu_{0}\right)$. The available information of the second Gegenbauer coefficient mainly comes from the QCD sum rules constructed from the correlation function with a light-ray tensor operator and a local vector current, which unfortunately give rise to the theory predictions sensitive to the choice of the input parameters. The crude estimate $b_{2}(1 \mathrm{GeV})=0.07 \pm 0.07$ from [85] will be used in our numerical analysis and an independent determination from the lattice QCD calculation will be very welcome in the future.

A natural choice of the factorization scale in the leading power factorization formula (2.50) is $\mu^{2}=\langle\bar{x}\rangle Q^{2}$ with $1 / 4 \leq\langle\bar{x}\rangle \leq 3 / 4$ corresponding to the characteristic virtuality of the intermediate quark displayed in figure $1(\mathrm{a})$, and it will be frozen at $\mu=1 \mathrm{GeV}$ for $\langle\bar{x}\rangle Q^{2}<1 \mathrm{GeV}^{2}$ at low $Q^{2}$ in order not to run into the nonperturbative QCD regime (see [8] for the discussion about the BLM proposal). Along similar lines, the factoriza- 

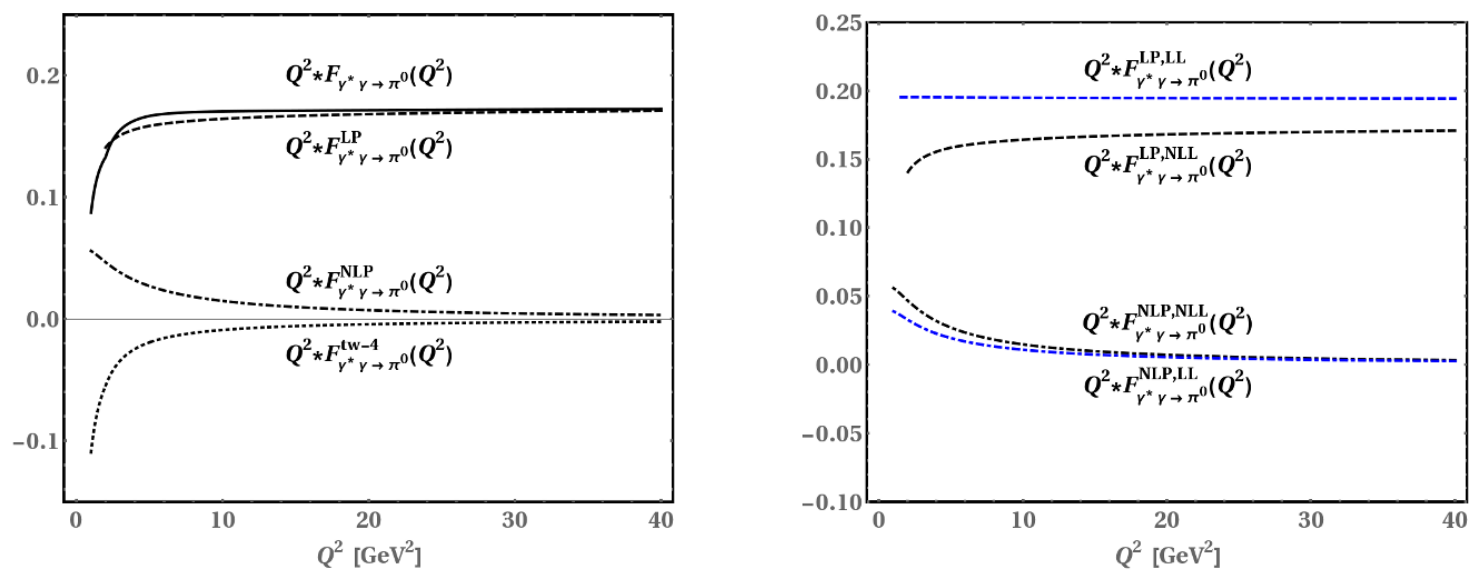

Figure 6. Left: distinct contributions to the $\pi^{0} \gamma^{*} \gamma$ form factor from the twist-two pion DA ("LP") with the BMS model at NLL, from the hadronic photon effect ("NLP") at NLL and from the twistfour pion DAs ("tw-4") at LO. The solid curve is obtained by adding up the above-mentioned three pieces together with the central inputs. Right: dependence of perturbative QCD corrections to the leading power contribution and to the hadronic photon effect with the BMS model, at LL and NLL accuracy, on the momentum transfer accessible at the BaBar and Belle experiments.

tion scale entering the NLL LCSRs for the hadronic photon effect (3.51) will be taken as $\mu^{2}=\langle x\rangle M^{2}+\langle\bar{x}\rangle Q^{2}$ as widely employed in the sum rule calculations [22].

Finally, the determination of the Borel mass $M^{2}$ and the threshold parameter $s_{0}$ can be achieved by applying the standard strategies described in $[88,89]$, and we can readily obtain

$$
M^{2}=(1.25 \pm 0.25) \mathrm{GeV}^{2}, \quad s_{0}=(0.70 \pm 0.05) \mathrm{GeV}^{2},
$$

in agreement with the intervals adopted in [90].

\subsection{Predictions for the $\pi^{0} \gamma^{*} \gamma$ form factor}

Now we will turn to investigate the phenomenological significance of distinct terms contributing to the pion-photon form factor. Taking the BMS model for the twist-two pion DA as an example, it is apparent from figure 6 that the twist-four correction and the hadronic photon contribution generate the destructive and constructive interference with the leading power effect (a similar observation for the high twist corrections already made in [22]) and there appears to be a strong cancellation between these two mechanisms in the whole $Q^{2} \leq 40 \mathrm{GeV}^{2}$ region. ${ }^{1}$ However, both subleading power effects become rapidly suppressed with the growing of the momentum transfer squared in contrast to the numerically sizeable soft power correction estimated from the dispersion approach [22]. Such discrepancy may be ascribed to the very definition of the "soft" effect in the formalism of [26], roughly corresponding to the $\rho$-resonance contribution to the $\pi^{0} \gamma^{*} \gamma$ form factor with the partonhadron duality approximation, which has no transparent counterpart in the framework of

\footnotetext{
${ }^{1}$ Both the twist-four correction and the hadronic photon effect are sizeable at $Q^{2} \sim[2,6] \mathrm{GeV}^{2}$, implying that the predicted $\pi^{0} \gamma^{*} \gamma$ form factor in the moderate $Q^{2}$ region should be taken cum grano salis, due to the yet unaccounted subleading power corrections, which can be potentially non-negligible at low $Q^{2}$.
} 

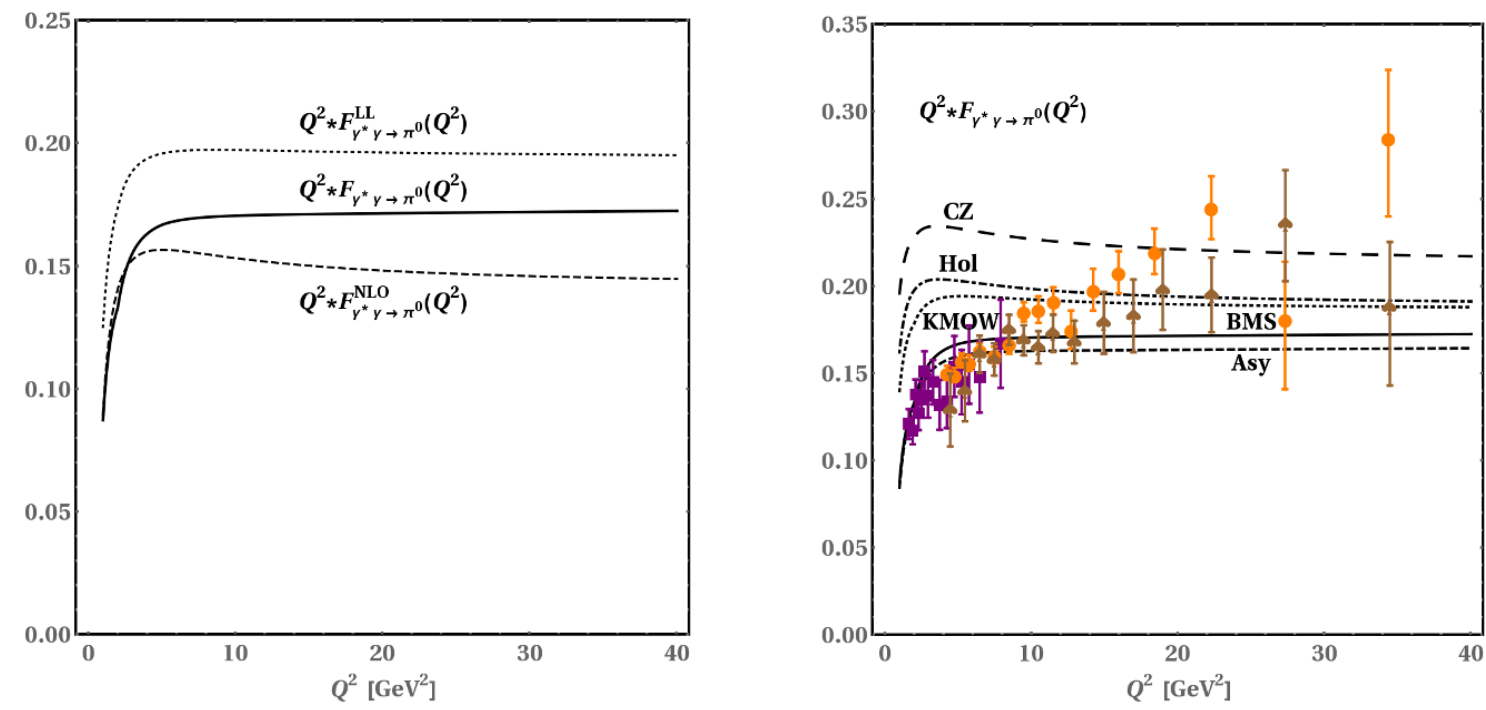

Figure 7. Left: the $Q^{2}$ dependence of the LL, NLO and NLL contributions to the $\pi^{0} \gamma^{*} \gamma$ form factor with the BMS model. Right: theory predictions for the pion-photon form factor with different models of the twist-two pion DA presented in (4.1). The experimental data are taken from CLEO [91] (purple squares), BaBar [21] (orange circles) and Belle [47] (brown spades).

perturbative QCD factorization. In addition, the NLL radiative corrections are observed to give rise to approximately $\mathcal{O}(15 \%)$ (almost $Q^{2}$-independent) shift to the LL predictions for both the leading power contribution and the hadronic photon effect.

To understand the phenomenological impact of the QCD resummation for the large logarithms appearing in the factorization formula for the leading power contribution and in the LCSRs for the hadronic photon correction, we further present in figure 7 our predictions for the $\pi^{0} \gamma^{*} \gamma$ form factor, at LL, NLO and NLL accuracy, with the BMS model. The NLO QCD corrections are found to induce $\mathcal{O}(25 \%)$ reduction of the tree-level results at $10 \mathrm{GeV}^{2} \leq Q^{2} \leq 40 \mathrm{GeV}^{2}$, however, the NLL resummation effect will enhance the NLO predictions by an amount of approximately $\mathcal{O}(10 \%)$, in accordance with the pattern for the perturbative QCD corrections observed in $[32,88]$. Inspecting the model dependence of pion-photon form factor on the leading twist pion DA displayed in figure 7 implies that the theory predictions with both the holographic and KMOW models can reasonably balance the BaBar and Belle data at high $Q^{2}$ without resorting to the "exotic" end-point behaviour as advocated in $[51,52]$. In fact, we have checked that the predicted $\pi^{0} \gamma^{*} \gamma$ form factor with the flat pion DA will overshoot both the BaBar and Belle data, in most $Q^{2}$ region of interest, at least in our framework. Given the fact that the end-point behaviour of the twisttwo pion DA in the holographic model differs from the standard postulation, motivated by the conformal expansion analysis, as employed for the KMOW model, we conclude that the local information of the pion DA cannot be extracted from the experimental measurements of the pion-photon form factor even in the leading power approximation. It needs further to be pointed out that our predictions with the holographic and KMOW models do not match the experimental data at $2 \mathrm{GeV}^{2} \leq Q^{2} \leq 8 \mathrm{GeV}^{2}$ well, where the 
power suppressed contributions from the yet higher-twist pion and photon DAs will become more pronounced and actually the large-momentum expansion applied for the construction of the factorization formula also becomes questionable. By contrast, the theory predictions from the dispersion approach $[22,23]$ can result in a satisfactory description of the BaBar and Belle data in the whole $Q^{2}$ region by introducing the nonperturbative modification of the QCD spectral density function. Moreover, it becomes apparent that the computed pionphoton form factor with the BMS model and the asymptotic pion DA are less favorable by the experimental measurements at high $Q^{2}$, albeit with the reasonable agreement achieved at low $Q^{2}$. Also, confronting the theory predictions from the $\mathrm{CZ}$ model with the BaBar and Belle data indicates a large value of the second Gegenbauber moment $a_{2}\left(\mu_{0}\right)$ is not favored, in agreement with the recent lattice QCD calculations [83, 92]. It is worthwhile to mention that the pion-photon transition form factor depends on the strong interaction dynamics encoded by the higher-twist pion DAs and the photon DAs (including the uncalculated effects from higher Fock states), hence measuring $F_{\gamma^{*} \gamma \rightarrow \pi^{0}}\left(Q^{2}\right)$ cannot be simply considered as a way of determining the higher Gegenbauer moments of the leading twist pion DA in the kinematic region accessible at the current experiments.

We present our final predictions for the $\pi^{0} \gamma^{*} \gamma$ form factor from the expression (3.52) with three different models of the twist-two pion DA in figure 8 , including the theory uncertainties due to the variations of the input parameters discussed before. To account for the yet higher-twist corrections discussed in the end of section 3, we have doubled the theory uncertainties of the twist- 4 term $F_{\gamma^{*} \gamma \rightarrow \pi^{0}}^{\mathrm{tw}-4}\left(Q^{2}\right)$ for a crude estimate. In addition, we assume that the missing NNLO QCD correction can be (partially) taken into account by varying the factorization scale $\mu$ in the interval as specified above, and a direct calculation of the two-loop hard function $T_{2}^{(2)}(x, \mu)$ from the operator matching equation (2.13) will be in demand for an unambiguous analysis. We further assigned $20 \%$ uncertainty for the first six nontrivial Gegenbauer coefficients of the holographic model in the numerical estimation for the illustration purpose. It turns out that the dominant theory uncertainties originate from the shape parameters of the leading twist pion and photon DAs instead of the variations of the factorization scales. Precision determinations of the higher Gegenbauer coefficients for both two DAs along the lines of $[83,92]$ will be essential to pin down the presently sizeable theory uncertainty in order to meet the challenge of the (potentially) more accurate experimental measurements at the BEPCII collider [93] and the SuperKEKB accelerator.

Concluding this section we make a few comments on the earlier QCD calculations of the pion-photon form factor in the literature.

- Applying the dispersion approach the subleading power "soft" contribution to the $\pi^{0} \gamma^{*} \gamma$ form factor was demonstrated in $[22,23]$ to be essential to accommodate the experimental data at low $Q^{2}$. However, the absence of the operator-level definition for the numerically prominent soft correction makes it difficult to trace back the corresponding dynamical mechanism in the SCET framework. It was further argued in $[22,23]$ that measuring the pion-photon form factor alone is not sufficient to determine the shape of the leading-twist pion DA unambiguously, in accordance with our observation made above. 


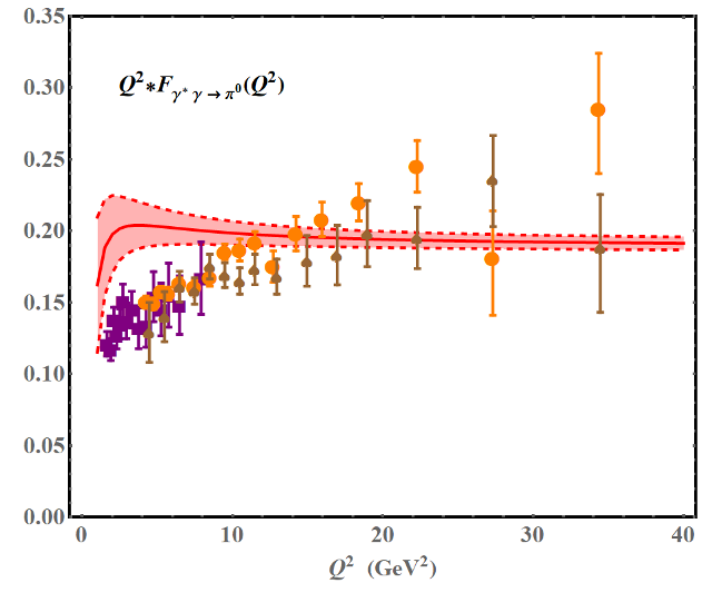

(a)

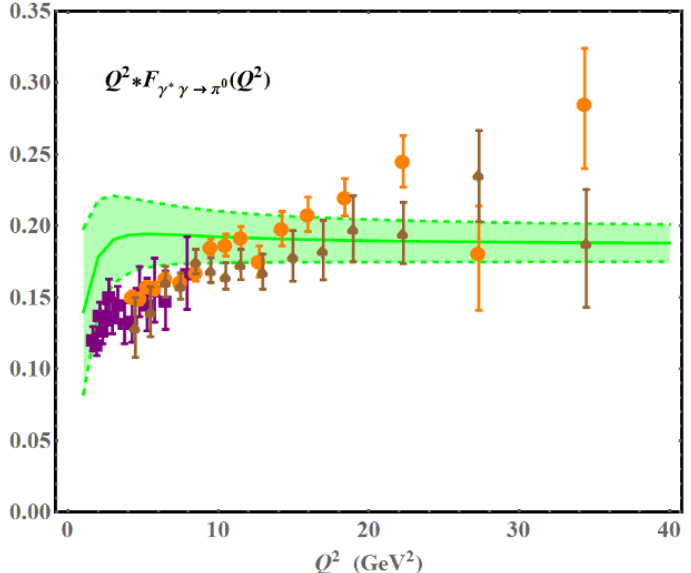

(b)

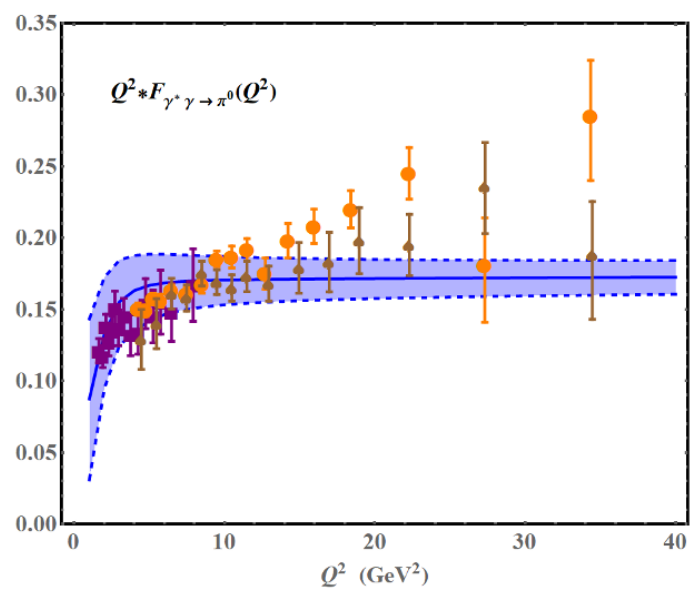

(c)

Figure 8. The $Q^{2}$ dependence of the $\pi^{0} \gamma^{*} \gamma$ form factor computed from (3.52) with (a) the holographic model, (b) the KMOW model, and (c) the BMS model. The shaded regions represent the combined theory uncertainties obtained by adding the separate errors in quadrature. The experimental data points from CLEO [91] (purple squares), BaBar [21] (orange circles) and Belle [47] (brown spades) are also displayed here.

- Implementing the threshold resummation for the enhanced logarithms $\left(\alpha_{s}(\mu) \ln x\right)^{n}$ in TMD factorization, the resulting theory predictions provide a reasonable description of the CLEO and BaBar data at $Q^{2} \leq 40 \mathrm{GeV}^{2}$ on account of the $Q^{2}$-dependent threshold function [94]. However, the importance of the small $x$ region would invalidate QCD factorization for the $\pi^{0} \gamma^{*} \gamma$ form factor, since the power counting scheme for the momentum fraction $x \sim \mathcal{O}(1)$ cannot be justified consequently.

- Employing the LCSR approach developed in [26], a systematic analysis of the $\pi^{0} \gamma^{*} \gamma$ form factor at $1 \mathrm{GeV}^{2} \leq Q^{2} \leq 5 \mathrm{GeV}^{2}$ with an emphasis on reducing the theoretical uncertainties from a wide variety of sources was carried out in [30], and the obtained 
theory predictions were found to comply with the available experimental data. Further development along this line can be achieved by working out a complete NNLO correction to the leading twist contribution and by performing an NLO computation for the twist-4 correction on the theoretical aspects.

\section{Conclusion}

Applying the standard OPE technique with the evanescent operator(s) we revisited the demonstration of QCD factorization for the pion-photon transition form factor at leading power in $1 / Q^{2}$ with both the NDR and HV schemes for $\gamma_{5}$ in the $D$-dimensional space. It has been shown explicitly at one loop that the renormalization scheme dependence of the short-distance matching coefficient and the twist-two pion DA are cancelled out precisely rendering the $\gamma_{5}$-prescription independence of the factorization formula for the leading power contribution to $F_{\gamma^{*} \gamma \rightarrow \pi^{0}}\left(Q^{2}\right)$. This can be readily understood from the fact that the QCD matrix element defined by two electromagnetic currents are free of the $\gamma_{5}$ ambiguity and the renormalization scheme dependence of the hard function arises from the infrared subtraction term completely. In the same vein, we established QCD factorization of the desired correlation function at one loop for the construction of the LCSRs for the hadronic photon contribution to the pion-photon form factor. By contrast, the corresponding QCD matrix element defined with an interpolating current for the pion and an electromagnetic current suffers from the $\gamma_{5}$ ambiguity, which can be resolved by applying the Ward identities of chiral gauge theories. The finite renormalization term introduced in the HV scheme to restore the appropriate Ward-Takahashi identities was found to provide the very transformation function to construct the hard matching coefficient in the NDR scheme. The NLL resummation of the parametrically large logarithms was also implemented by solving the relevant two-loop evolution equations in momentum space.

Taking into account the leading power contribution and the hadronic photon effect at NLL as well as the twist-four correction from the pion DAs at tree level, we further explored the phenomenological consequence of the perturbative QCD corrections and the subleading power contributions. Interestingly, the observed strong cancellation between the two power suppressed mechanisms leads to the insignificant correction to the leading power contribution (almost) in the whole $Q^{2}$ region accessible at the current experiments. In addition, we paid a particular attention to the model dependence of the theory predictions for $F_{\gamma^{*} \gamma \rightarrow \pi^{0}}\left(Q^{2}\right)$ on the twist-two pion DA. Both the holographic and KMOW models turned out to balance the BaBar and Belle data reasonably well at high $Q^{2}$, despite the visible discrepancy at low $Q^{2}$ which could be compensated by the unaccounted subleading power corrections of both perturbative and nonperturbative origins. It was also demonstrated that the end-point behaviour of the pion DA cannot be extracted by matching the theory predictions for the pion-photon form factor with the experimental measurements.

Aiming at a better confrontation with the BaBar and Belle data, further improvements of our calculations can be made by first carrying out the perturbative correction to the twist- 4 contribution from both the two-particle and three-particle pion DAs, which is also of conceptual interest in the framework of perturbative QCD factorization, and then 
by evaluating the high twist contributions from the photon DAs with the LCSR approach. Phenomenological applications of the techniques discussed in this work can be also pursued in the context of the $\gamma^{*} \gamma \rightarrow\left(\eta^{(\prime)}, \eta_{c}\right)$ transition form factors [33, 95] for understanding the quark-gluon structure of eta mesons and heavy quarkonium states, the radiative leptonic $B$-meson decays for the determination of the inverse moment $\lambda_{B}$, the radiative penguin decays of $B$-mesons for the precision test of the quark-flavour structure of the Standard Model, and the radiative heavy-hadron decays for constraining the magnetic susceptibility of the quark condensate [86]. To conclude, the anatomy of the subleading power contributions for the exclusive hadronic reactions is of high interest for understanding the general structures of the large momentum/mass expansion in QCD and for hunting new physics in the quark-flavour sector as indicated by the various flavour "anomalies" observed at the ongoing experiments.

\section{Acknowledgments}

We are grateful to Martin Beneke and Vladimir Braun for illuminating discussions and to Vladimir Braun for many valuable comments on the manuscript. Y.M.W acknowledges support from the National Youth Thousand Talents Program, the Youth Hundred Academic Leaders Program of Nankai University, and the NSFC with Grant No. 11675082. The work of Y.L.S is supported by Natural Science Foundation of Shandong Province, China under Grant No. ZR2015AQ006.

\section{A Two-loop evolution functions}

\section{A.1 RG evolution of the twist-2 pion DA at two loops}

We first collect the explicit expressions of the RG functions $E_{V, n}^{\mathrm{NLO}}$ and $d_{V, n}^{k}$ appeared in the two-loop evolution matrix of the twist-2 pion DA, following closely [22]. Our conventions for the QCD beta-function and the anomalous dimensions of the local conformal operator [63]

$$
O_{V, k}(\mu)=(i \bar{n} \cdot \partial)^{k} \bar{q}(0) \not h \gamma_{5} C_{k}^{3 / 2}(\bar{n} \cdot \stackrel{\leftrightarrow}{D} / \bar{n} \cdot \partial) q(0)
$$

are given by

$$
\begin{aligned}
& \mu \frac{d \alpha_{s}(\mu)}{d \mu}=\beta\left(\alpha_{s}\right)=-2 \alpha_{s} \sum_{n=0} \beta_{n}\left(\frac{\alpha_{s}}{4 \pi}\right)^{n+1} \\
& \gamma_{V, n}\left(\alpha_{s}\right)=-\sum_{n=0} \gamma_{V, n}^{(0)}\left(\frac{\alpha_{s}}{4 \pi}\right)^{n+1} .
\end{aligned}
$$

The first three perturbative coefficients of $\beta_{n}$ are

$$
\beta_{0}=11-\frac{2 N_{f}}{3}, \quad \beta_{1}=102-\frac{38 N_{f}}{3}, \quad \beta_{2}=\frac{2857}{2}-\frac{5033 N_{f}}{18}+\frac{325 N_{f}^{2}}{54},
$$

and the well-known LO anomalous dimension $\gamma_{V, n}^{(0)}$ reads

$$
\gamma_{V, n}^{(0)}=2 C_{F}\left(1-\frac{2}{(n+1)(n+2)}+4 \sum_{k=2}^{n+1} \frac{1}{k}\right) \text {. }
$$


The NLO anomalous dimension $\gamma_{V, n}^{(1)}$ can be obtained from the convolution integral

$$
\gamma_{V, n}^{(1)}=-\frac{8(2 n+3)}{(n+1)(n+2)} \int_{0}^{1} d x \int_{0}^{1} d y\left[V_{1}(x, y)\right]_{+} y \bar{y}\left[C_{n}^{3 / 2}(2 x-1)\right]^{2} .
$$

Making use of the harmonic sums [96, 97]

$$
S_{l}(n)=\sum_{k=1}^{n} \frac{1}{k^{l}}, \quad S_{l}^{\prime}(n)=2^{l-1} \sum_{k=1}^{n}\left[1+(-1)^{k}\right] \frac{1}{k^{l}}, \quad \tilde{S}(n)=\sum_{k=1}^{n} \frac{(-1)^{k}}{k^{2}} S_{1}(k),
$$

the above-mentioned integral (A.6) can be further computed as [98]

$$
\begin{aligned}
\gamma_{V, n}^{(1)}= & 4\left(C_{F}^{2}-\frac{1}{2} C_{F} C_{A}\right)\left\{\frac{4(2 n+3)}{(n+1)^{2}(n+2)^{2}} S_{1}(n+1)-2 \frac{3 n^{3}+10 n^{2}+11 n+3}{(n+1)^{3}(n+2)^{3}}\right. \\
& +4\left(2 S_{1}(n+1)-\frac{1}{(n+1)(n+2)}\right)\left(S_{2}(n+1)-S_{2}^{\prime}(n+1)\right)+16 \tilde{S}(n+1) \\
& \left.+6 S_{2}(n+1)-\frac{3}{4}-2 S_{3}^{\prime}(n+1)-4(-1)^{n+1} \frac{2 n^{2}+6 n+5}{(n+1)^{3}(n+2)^{3}}\right\} \\
& +4 C_{F} C_{A}\left\{S_{1}(n+1)\left(\frac{134}{9}+\frac{2(2 n+3)}{(n+1)^{2}(n+2)^{2}}\right)-4 S_{1}(n+1) S_{2}(n+1)\right. \\
& +S_{2}(n+1)\left(-\frac{13}{3}+\frac{2}{(n+1)(n+2)}\right)-\frac{43}{24} \\
& \left.-\frac{1}{9} \frac{151 n^{4}+867 n^{3}+1792 n^{2}+1590 n+523}{(n+1)^{3}(n+2)^{3}}\right\} \\
& +2 C_{F} N_{f}\left\{-\frac{40}{9} S_{1}(n+1)+\frac{8}{3} S_{2}(n+1)+\frac{1}{3}+\frac{4}{9} \frac{11 n^{2}+27 n+13}{(n+1)^{2}(n+2)^{2}}\right\} .
\end{aligned}
$$

According the master solutions displayed in (53) and (54) of [63] and comparing with (2.49), we can readily find that $[22]$

$$
\begin{aligned}
E_{V, n}^{\mathrm{LO}}\left(\mu, \mu_{0}\right) & =\left(\frac{\alpha_{s}(\mu)}{\alpha_{s}\left(\mu_{0}\right)}\right)^{\gamma_{V, n}^{(0)} /\left(2 \beta_{0}\right)}, \\
E_{V, n}^{\mathrm{NLO}}\left(\mu, \mu_{0}\right) & =E_{V, n}^{\mathrm{LO}}\left(\mu, \mu_{0}\right)\left\{1+\frac{\alpha_{s}(\mu)-\alpha_{s}\left(\mu_{0}\right)}{8 \pi} \frac{\gamma_{V, n}^{(0)}}{\beta_{0}}\left(\frac{\gamma_{V, n}^{(1)}}{\gamma_{V, n}^{(0)}}-\frac{\beta_{1}}{\beta_{0}}\right)\right\},
\end{aligned}
$$

and the off-diagonal evolution coefficient $d_{V, n}^{k}$ reads

$$
d_{V, n}^{k}=\frac{M_{V, n}^{k}}{\gamma_{V, n}^{(0)}-\gamma_{V, k}^{(0)}-2 \beta_{0}}\left[1-\left(\frac{\alpha_{s}(\mu)}{\alpha_{s}\left(\mu_{0}\right)}\right)^{\left(\gamma_{V, n}^{(0)}-\gamma_{V, k}^{(0)}-2 \beta_{0}\right) /\left(2 \beta_{0}\right)}\right] .
$$

The matrix element $M_{V, n}^{k}$ is given by

$$
\begin{aligned}
M_{V, n}^{k}= & \frac{(k+1)(k+2)(2 n+3)}{(n+1)(n+2)}\left[\gamma_{V, n}^{(0)}-\gamma_{V, k}^{(0)}\right]\left\{\frac{8 C_{F} A_{n}^{k}-\gamma_{V, k}^{(0)}-2 \beta_{0}}{(n-k)(n+k+3)}\right. \\
& \left.+4 C_{F} \frac{A_{n}^{k}-\psi(n+2)+\psi(1)}{(k+1)(k+2)}\right\},
\end{aligned}
$$


with

$$
\begin{aligned}
A_{n}^{k} & =\psi\left(\frac{n+k+4}{2}\right)-\psi\left(\frac{n-k}{2}\right)+2 \psi(n-k)-\psi(n+2)-\psi(1), \\
\psi(z) & =d \ln \Gamma(z) / d z .
\end{aligned}
$$

\section{A.2 RG evolution of the twist-2 photon DA at two loops}

Along the lines of the discussion for the pion DA, we first need the anomalous dimensions of the following conformal operator

$$
O_{T, k}^{\nu}(\mu)=(i n \cdot \partial)^{k} \bar{q}(0) \not h \gamma^{\nu, \perp} C_{k}^{3 / 2}(n \cdot \stackrel{\leftrightarrow}{D} / n \cdot \partial) q(0)
$$

which can be perturbatively expanded in QCD

$$
\gamma_{T, n}\left(\alpha_{s}\right)=-\sum_{n=0} \gamma_{T, n}^{(0)}\left(\frac{\alpha_{s}}{4 \pi}\right)^{n+1}
$$

The one-loop anomalous dimension $\gamma_{T, n}^{(0)}$ is given by $[68,70]$

$$
\gamma_{T, n}^{(0)}=2 C_{F}\left(1+4 \sum_{k=2}^{n+1} \frac{1}{k}\right),
$$

and the NLO anomalous dimension $\gamma_{T, n}^{(1)}$ can be extracted from the two-loop splitting function for the twist-2 transversity distribution in deep-inelastic scattering (DIS) [98-100]

$$
\begin{aligned}
\gamma_{T, n}^{(1)}= & 4 C_{F}^{2}\left[-\frac{1}{4}-2 S_{1}(n+1)+S_{2}(n+1)\right]+\frac{16}{9} N_{f} C_{F}\left[\frac{3}{8}-5 S_{1}(n+1)+3 S_{2}(n+1)\right] \\
& +C_{A} C_{F}\left[-\frac{20}{3}+\frac{572}{9} S_{1}(n+1)-\frac{58}{3} S_{2}(n+1)-16 S_{1}(n+1) S_{2}(n+1)\right] \\
& -8 C_{F}\left(C_{F}-\frac{1}{2} C_{A}\right)\left\{\frac{1}{4}+\frac{1+(-1)^{n}}{(n+1)(n+2)}-\frac{5}{2} S_{2}(n+1)+S_{3}^{\prime}(n+1)\right. \\
& \left.-8 \tilde{S}(n+1)-S_{1}(n+1)\left[1+4 S_{2}(n+1)-4 S_{2}^{\prime}(n+1)\right]\right\} .
\end{aligned}
$$

The explicit expressions of the RG functions $E_{T, n}^{\mathrm{NLO}}$ and $d_{T, n}^{k}$ can be obtained from that of $E_{V, n}^{\mathrm{NLO}}$ and $d_{V, n}^{k}$ given above with the replacement rule $\gamma_{V, n}^{(i)} \rightarrow \gamma_{T, n}^{(i)}(i=0,1)[63,67]$.

\section{B Spectral representations}

We present the dispersion representations of convolution integrals entering the NLL QCD factorization formula (3.32) in order to construct the sum rules for the hadronic photon correction to the pion-photon form factor. We have verified the spectral representations in what follows numerically by checking the corresponding dispersion integrals.

$$
\begin{aligned}
& \frac{1}{\pi} \operatorname{Im}_{s} \int_{0}^{1} d u \frac{1}{u r+\bar{u}} \frac{u r-\bar{u}}{u \bar{u} \bar{r}} \ln (u r+\bar{u}) \phi_{\gamma}(u, \mu) \\
& =\frac{Q^{2}}{Q^{2}+s} \int_{0}^{1} d u \theta\left(u-\frac{Q^{2}}{Q^{2}+s}\right)\left[\frac{\bar{u}-u}{u \bar{u}}+2 \ln \left(\frac{u s-\bar{u} Q^{2}}{Q^{2}}\right) \frac{d}{d u}\right] \phi_{\gamma}(u, \mu) .
\end{aligned}
$$




$$
\begin{aligned}
& \frac{1}{\pi} \operatorname{Im}_{s} \int_{0}^{1} d u \frac{1}{u r+\bar{u}} \frac{u r-\bar{u}}{u \bar{u} \bar{r}} \ln ^{2}(u r+\bar{u}) \phi_{\gamma}(u, \mu) \\
& =\frac{Q^{2}}{Q^{2}+s} \int_{0}^{1} d u \theta\left(u-\frac{Q^{2}}{Q^{2}+s}\right)\left\{\left[2 \ln ^{2}\left(\frac{u s-\bar{u} Q^{2}}{Q^{2}}\right)-\frac{2 \pi^{2}}{3}\right] \frac{d}{d u}\right. \\
& \left.\quad+2 \frac{\bar{u}-u}{u \bar{u}} \ln \left(\frac{u s-\bar{u} Q^{2}}{Q^{2}}\right)\right\} \phi_{\gamma}(u, \mu) .
\end{aligned}
$$

$\frac{1}{\pi} \operatorname{Im}_{s} \int_{0}^{1} d u \frac{1}{u r+\bar{u}} \frac{u r-\bar{u}}{u \bar{u} \bar{r}} \ln (u r+\bar{u}) \ln r \phi_{\gamma}(u, \mu)$

$=\frac{Q^{2}}{Q^{2}+s} \int_{0}^{1} d u\left\{\frac{\bar{u}-u}{u \bar{u}}\left[\theta\left(u-\frac{Q^{2}}{Q^{2}+s}\right) \ln \left(\frac{s}{Q^{2}}\right)+\ln \left|\frac{u s-\bar{u} Q^{2}}{Q^{2}}\right|\right] \phi_{\gamma}(u, \mu)\right.$

$$
+\left[\ln ^{2}\left|\frac{u s-\bar{u} Q^{2}}{Q^{2}}\right|+\theta\left(u-\frac{Q^{2}}{Q^{2}+s}\right)\left(2 \ln \left(\frac{u s-\bar{u} Q^{2}}{Q^{2}}\right) \ln \left(\frac{s}{Q^{2}}\right)-\pi^{2}\right)\right]
$$$$
\left.\times \frac{d}{d u} \phi_{\gamma}(u, \mu)\right\} \text {. }
$$

$\frac{1}{\pi} \operatorname{Im}_{s} \int_{0}^{1} d u \frac{1}{u r+\bar{u}} \frac{r}{\bar{u} \bar{r}} \ln r \phi_{\gamma}(u, \mu)$

$=-\frac{Q^{2}}{Q^{2}+s} \ln \left(\frac{s}{Q^{2}}\right) \phi_{\gamma}\left(\frac{Q^{2}}{Q^{2}+s}, \mu\right)-\int_{0}^{1} \frac{d u}{\bar{u}}\left[\frac{Q^{2}}{s+Q^{2}}+\mathcal{P} \frac{\bar{u} Q^{2}}{u s-\bar{u} Q^{2}}\right] \phi_{\gamma}(u, \mu)$.

$\frac{1}{\pi} \operatorname{Im}_{s} \int_{0}^{1} d u \frac{1}{u r+\bar{u}} \frac{r}{\bar{u} \bar{r}} \ln (u r+\bar{u}) \ln r \phi_{\gamma}(u, \mu)$

$=\frac{Q^{2}}{Q^{2}+s} \int_{0}^{1} d u\left\{-\frac{1}{\bar{u}}\left[\theta\left(u-\frac{Q^{2}}{Q^{2}+s}\right) \ln \left(\frac{s}{Q^{2}}\right)+\ln \left|\frac{u s-\bar{u} Q^{2}}{Q^{2}}\right|\right] \phi_{\gamma}(u, \mu)\right.$

$+\frac{1}{2}\left[\ln ^{2}\left|\frac{u s-\bar{u} Q^{2}}{Q^{2}}\right|+\theta\left(u-\frac{Q^{2}}{Q^{2}+s}\right)\left(2 \ln \left(\frac{u s-\bar{u} Q^{2}}{Q^{2}}\right) \ln \left(\frac{s}{Q^{2}}\right)-\pi^{2}\right)\right]$

$\left.\times \frac{d}{d u} \phi_{\gamma}(u, \mu)\right\}$.

$\frac{1}{\pi} \operatorname{Im}_{s} \int_{0}^{1} d u \frac{1}{u r+\bar{u}} \frac{r}{\bar{u} \bar{r}} \ln ^{2} r \phi_{\gamma}(u, \mu)$

$=-\frac{Q^{2}}{Q^{2}+s}\left[\ln ^{2}\left(\frac{s}{Q^{2}}\right)-\pi^{2}\right] \phi_{\gamma}\left(\frac{Q^{2}}{Q^{2}+s}, \mu\right)$

$$
-2 \ln \left(\frac{s}{Q^{2}}\right) \int_{0}^{1} \frac{d u}{\bar{u}}\left[\frac{Q^{2}}{Q^{2}+s}+\mathcal{P} \frac{\bar{u} Q^{2}}{u s-\bar{u} Q^{2}}\right] \phi_{\gamma}(u, \mu) .
$$

$\frac{1}{\pi} \operatorname{Im}_{s} \int_{0}^{1} d u \frac{1}{u r+\bar{u}} \frac{1}{u \bar{r}}(\ln r+3) \ln (\bar{u}+u r) \phi_{\gamma}(u, \mu)$

$=\frac{Q^{2}}{Q^{2}+s} \int_{0}^{1} d u\left\{-\frac{\phi_{\gamma}(u, \mu)}{u}\left[\theta\left(u-\frac{Q^{2}}{Q^{2}+s}\right)\left(\ln \left(\frac{s}{Q^{2}}\right)+3\right)+\ln \left|\frac{u s-\bar{u} Q^{2}}{Q^{2}}\right|\right]\right.$

$-\frac{1}{2}\left[\ln ^{2}\left|\frac{u s-\bar{u} Q^{2}}{Q^{2}}\right|+\theta\left(u-\frac{Q^{2}}{Q^{2}+s}\right)\left(\left(2 \ln \left(\frac{s}{Q^{2}}\right)+3\right) \ln \left(\frac{u s-\bar{u} Q^{2}}{Q^{2}}\right)-\pi^{2}\right)\right]$ $\left.\times \frac{d}{d u} \phi_{\gamma}(u, \mu)\right\}$. 
Here, the parameter $p^{2}$ in the definition of $r$ should be understood as $s$ in the above convolution integrals and $\mathcal{P}$ represents the principle-value prescription.

Open Access. This article is distributed under the terms of the Creative Commons Attribution License (CC-BY 4.0), which permits any use, distribution and reproduction in any medium, provided the original author(s) and source are credited.

\section{References}

[1] G.P. Lepage and S.J. Brodsky, Exclusive Processes in Perturbative Quantum Chromodynamics, Phys. Rev. D 22 (1980) 2157 [InSPIRE].

[2] A.V. Efremov and A.V. Radyushkin, Factorization and Asymptotical Behavior of Pion Form-Factor in QCD, Phys. Lett. B 94 (1980) 245 [inSPIRE].

[3] A. Duncan and A.H. Mueller, Asymptotic Behavior of Exclusive and Almost Exclusive Processes, Phys. Lett. B 90 (1980) 159 [inSPIRE].

[4] I.Z. Rothstein, Factorization, power corrections and the pion form-factor, Phys. Rev. D 70 (2004) 054024 [hep-ph/0301240] [INSPIRE].

[5] F. del Aguila and M.K. Chase, Higher Order QCD Corrections To Exclusive Two Photon Processes, Nucl. Phys. B 193 (1981) 517 [InSPIRE].

[6] E. Braaten, QCD Corrections To Meson-Photon Transition Form-Factors, Phys. Rev. D 28 (1983) 524 [INSPIRE].

[7] E.P. Kadantseva, S.V. Mikhailov and A.V. Radyushkin, Total $\alpha^{-} s$ Corrections to Processes $\gamma^{*} \gamma^{*} \rightarrow \pi^{0}$ and $\gamma^{*} \pi \rightarrow \pi$ in a Perturbative QCD, Yad. Fiz. 44 (1986) 507 [Sov. J. Nucl. Phys. 44 (1986) 326] [INSPIRE].

[8] B. Melic, D. Mueller and K. Passek-Kumericki, Next-to-next-to-leading prediction for the photon to pion transition form-factor, Phys. Rev. D 68 (2003) 014013 [hep-ph/0212346] [INSPIRE].

[9] G. Bonneau, Some Fundamental but Elementary Facts on Renormalization and Regularization: A Critical Review of the Eighties, Int. J. Mod. Phys. A 5 (1990) 3831 [INSPIRE].

[10] J.C. Collins, Renormalization: An Introduction to Renormalization, The Renormalization Group, and the Operator Product Expansion, Cambridge University Press, (1984).

[11] S.A. Larin, The renormalization of the axial anomaly in dimensional regularization, Phys. Lett. B 303 (1993) 113 [hep-ph/9302240] [INSPIRE].

[12] C.P. Martin and D. Sánchez-Ruiz, Action principles, restoration of BRS symmetry and the renormalization group equation for chiral nonAbelian gauge theories in dimensional renormalization with a nonanticommuting $\gamma_{5}$, Nucl. Phys. B 572 (2000) 387 [hep-th/9905076] [INSPIRE].

[13] F. Jegerlehner, Facts of life with $\gamma_{5}$, Eur. Phys. J. C 18 (2001) 673 [hep-th/0005255] [INSPIRE].

[14] S. Moch, J.A.M. Vermaseren and A. Vogt, $O n \gamma_{5}$ in higher-order QCD calculations and the NNLO evolution of the polarized valence distribution, Phys. Lett. B 748 (2015) 432 [arXiv: 1506. 04517] [INSPIRE]. 
[15] D. Gutiérrez-Reyes, I. Scimemi and A.A. Vladimirov, Twist-2 matching of transverse momentum dependent distributions, Phys. Lett. B 769 (2017) 84 [arXiv:1702.06558] [INSPIRE].

[16] M. Beneke, Y. Kiyo and D.s. Yang, Loop corrections to subleading heavy quark currents in SCET, Nucl. Phys. B 692 (2004) 232 [hep-ph/0402241] [INSPIRE].

[17] M. Beneke and D. Yang, Heavy-to-light B meson form-factors at large recoil energy: Spectator-scattering corrections, Nucl. Phys. B 736 (2006) 34 [hep-ph/0508250] [INSPIRE].

[18] M. Beneke and S. Jager, Spectator scattering at NLO in non-leptonic B decays: Tree amplitudes, Nucl. Phys. B 751 (2006) 160 [hep-ph/0512351] [INSPIRE].

[19] M.J. Dugan and B. Grinstein, On the vanishing of evanescent operators, Phys. Lett. B 256 (1991) 239 [INSPIRE].

[20] S. Herrlich and U. Nierste, Evanescent operators, scheme dependences and double insertions, Nucl. Phys. B 455 (1995) 39 [hep-ph/9412375] [INSPIRE].

[21] BABAR collaboration, B. Aubert et al., Measurement of the $\gamma \gamma * \rightarrow \pi^{0}$ transition form factor, Phys. Rev. D 80 (2009) 052002 [arXiv:0905.4778] [INSPIRE].

[22] S.S. Agaev, V.M. Braun, N. Offen and F.A. Porkert, Light Cone Sum Rules for the $\pi^{0} \gamma * \gamma$ Form Factor Revisited, Phys. Rev. D 83 (2011) 054020 [arXiv: 1012.4671] [INSPIRE].

[23] S.S. Agaev, V.M. Braun, N. Offen and F.A. Porkert, BELLE Data on the $\pi^{0} \gamma * \gamma$ Form Factor: A Game Changer?, Phys. Rev. D 86 (2012) 077504 [arXiv:1206.3968] [INSPIRE].

[24] P. Kroll, The form factors for the photon to pseudoscalar meson transitions - an update, Eur. Phys. J. C 71 (2011) 1623 [arXiv:1012.3542] [inSPIRE].

[25] H.-N. Li, Y.-L. Shen and Y.-M. Wang, Joint resummation for pion wave function and pion transition form factor, JHEP 01 (2014) 004 [arXiv:1310.3672] [INSPIRE].

[26] A. Khodjamirian, Form-factors of $\gamma * \rho \rightarrow \pi$ and $\gamma * \gamma \rightarrow \pi^{0}$ transitions and light cone sum rules, Eur. Phys. J. C 6 (1999) 477 [hep-ph/9712451] [INSPIRE].

[27] N.G. Stefanis, A.P. Bakulev, S.V. Mikhailov and A.V. Pimikov, Can We Understand an Auxetic Pion-Photon Transition Form Factor within QCD?, Phys. Rev. D 87 (2013) 094025 [arXiv: 1202.1781] [INSPIRE].

[28] A.P. Bakulev, S.V. Mikhailov, A.V. Pimikov and N.G. Stefanis, Pion-photon transition: The new QCD frontier, Phys. Rev. D 84 (2011) 034014 [arXiv: 1105.2753] [InSPIRE].

[29] A.P. Bakulev, S.V. Mikhailov, A.V. Pimikov and N.G. Stefanis, Comparing antithetic trends of data for the pion-photon transition form factor, Phys. Rev. D 86 (2012) 031501 [arXiv: 1205.3770] [INSPIRE].

[30] S.V. Mikhailov, A.V. Pimikov and N.G. Stefanis, Systematic estimation of theoretical uncertainties in the calculation of the pion-photon transition form factor using light-cone sum rules, Phys. Rev. D 93 (2016) 114018 [arXiv:1604.06391] [INSPIRE].

[31] V.M. Braun and A. Khodjamirian, Soft contribution to $B \rightarrow \gamma \ell \nu_{\ell}$ and the B-meson distribution amplitude, Phys. Lett. B 718 (2013) 1014 [arXiv:1210.4453] [INSPIRE].

[32] Y.-M. Wang, Factorization and dispersion relations for radiative leptonic B decay, JHEP 09 (2016) 159 [arXiv:1606.03080] [INSPIRE]. 
[33] S.S. Agaev, V.M. Braun, N. Offen, F.A. Porkert and A. Schäfer, Transition form factors $\gamma^{*} \gamma \rightarrow \eta$ and $\gamma^{*} \gamma \rightarrow \eta^{\prime}$ in QCD, Phys. Rev. D 90 (2014) 074019 [arXiv:1409.4311] [INSPIRE].

[34] V.M. Braun, N. Kivel, M. Strohmaier and A.A. Vladimirov, Electroproduction of tensor mesons in QCD, JHEP 06 (2016) 039 [arXiv:1603.09154] [INSPIRE].

[35] P. Ball, V.M. Braun and N. Kivel, Photon distribution amplitudes in QCD, Nucl. Phys. B 649 (2003) 263 [hep-ph/0207307] [INSPIRE].

[36] H.-n. Li and G.F. Sterman, The perturbative pion form-factor with Sudakov suppression, Nucl. Phys. B 381 (1992) 129 [InSPIRE].

[37] S. Nandi and H.-n. Li, Next-to-leading-order corrections to exclusive processes in $k_{T}$ factorization, Phys. Rev. D 76 (2007) 034008 [arXiv: 0704.3790] [INSPIRE].

[38] I.V. Musatov and A.V. Radyushkin, Transverse momentum and Sudakov effects in exclusive QCD processes: $\gamma * \gamma \pi^{0}$ form-factor, Phys. Rev. D 56 (1997) 2713 [hep-ph/9702443] [INSPIRE].

[39] X.-G. Wu and T. Huang, An Implication on the Pion Distribution Amplitude from the Pion-Photon Transition Form Factor with the New BABAR Data, Phys. Rev. D 82 (2010) 034024 [arXiv: 1005. 3359] [inSPIRE].

[40] Y.-C. Chen and H.-n. Li, Three-parton contribution to pion form factor in $k_{T}$ factorization, Phys. Rev. D 84 (2011) 034018 [arXiv: 1104.5398] [INSPIRE].

[41] H.-n. Li and Y.-M. Wang, Non-dipolar Wilson links for transverse-momentum-dependent wave functions, JHEP 06 (2015) 013 [arXiv: 1410.7274] [INSPIRE].

[42] X.-G. He, T. Li, X.-Q. Li and Y.-M. Wang, PQCD calculation for $\Lambda_{b} \rightarrow \Lambda \gamma$ in the standard model, Phys. Rev. D 74 (2006) 034026 [hep-ph/0606025] [INSPIRE].

[43] C.-D. Lu, Y.-M. Wang, H. Zou, A. Ali and G. Kramer, Anatomy of the pQCD Approach to the Baryonic Decays $\Lambda_{b} \rightarrow p \pi, p K$, Phys. Rev. D 80 (2009) 034011 [arXiv:0906.1479] [INSPIRE].

[44] H.-n. Li, Y.-L. Shen, Y.-M. Wang and H. Zou, Next-to-leading-order correction to pion form factor in $k_{T}$ factorization, Phys. Rev. D 83 (2011) 054029 [arXiv:1012.4098] [INSPIRE].

[45] H.-n. Li, Y.-L. Shen and Y.-M. Wang, Next-to-leading-order corrections to $B \rightarrow \pi$ form factors in $k_{T}$ factorization, Phys. Rev. D 85 (2012) 074004 [arXiv:1201.5066] [INSPIRE].

[46] H.-N. Li, Y.-L. Shen and Y.-M. Wang, Resummation of rapidity logarithms in B meson wave functions, JHEP 02 (2013) 008 [arXiv: 1210.2978] [INSPIRE].

[47] Belle collaboration, S. Uehara et al., Measurement of $\gamma \gamma^{*} \rightarrow \pi^{0}$ transition form factor at Belle, Phys. Rev. D 86 (2012) 092007 [arXiv:1205.3249] [inSPIRE].

[48] P. Masjuan, $\gamma * \gamma \rightarrow \pi^{0}$ transition form factor at low-energies from a model-independent approach, Phys. Rev. D 86 (2012) 094021 [arXiv:1206.2549] [INSPIRE].

[49] M. Hoferichter, B. Kubis, S. Leupold, F. Niecknig and S.P. Schneider, Dispersive analysis of the pion transition form factor, Eur. Phys. J. C 74 (2014) 3180 [arXiv:1410.4691] [INSPIRE].

[50] A. Gérardin, H.B. Meyer and A. Nyffeler, Lattice calculation of the pion transition form factor $\pi^{0} \rightarrow \gamma^{*} \gamma^{*}$, Phys. Rev. D 94 (2016) 074507 [arXiv:1607.08174] [INSPIRE]. 
[51] A.V. Radyushkin, Shape of Pion Distribution Amplitude, Phys. Rev. D 80 (2009) 094009 [arXiv:0906.0323] [INSPIRE].

[52] M.V. Polyakov, On the Pion Distribution Amplitude Shape, JETP Lett. 90 (2009) 228 [arXiv:0906.0538] [INSPIRE].

[53] Y. Matiounine, J. Smith and W.L. van Neerven, Two loop operator matrix elements calculated up to finite terms for polarized deep inelastic lepton-hadron scattering, Phys. Rev. D 58 (1998) 076002 [hep-ph/9803439] [INSPIRE].

[54] V. Ravindran, J. Smith and W.L. van Neerven, NNLO corrections to massive lepton pair production in longitudinally polarized proton proton collisions, Nucl. Phys. B 682 (2004) 421 [hep-ph/0311304] [INSPIRE].

[55] M. Beneke and V.A. Smirnov, Asymptotic expansion of Feynman integrals near threshold, Nucl. Phys. B 522 (1998) 321 [hep-ph/9711391] [INSPIRE].

[56] B. Melic, B. Nizic and K. Passek, BLM scale setting for the pion transition form-factor, Phys. Rev. D 65 (2002) 053020 [hep-ph/0107295] [INSPIRE].

[57] M.H. Sarmadi, The Asymptotic Pion Form-factor Beyond the Leading Order, Phys. Lett. B 143 (1984) 471 [INSPIRE].

[58] F.M. Dittes and A.V. Radyushkin, Two Loop Contribution To The Evolution Of The Pion Wave Function, Phys. Lett. B 134 (1984) 359 [INSPIRE].

[59] G.R. Katz, Two Loop Feynman Gauge Calculation of the Meson Nonsinglet Evolution Potential, Phys. Rev. D 31 (1985) 652 [inSPIRE].

[60] S.V. Mikhailov and A.V. Radyushkin, Evolution Kernels in QCD: Two Loop Calculation in Feynman Gauge, Nucl. Phys. B 254 (1985) 89 [INSPIRE].

[61] A.V. Belitsky, D. Mueller and A. Freund, Reconstruction of nonforward evolution kernels, Phys. Lett. B 461 (1999) 270 [hep-ph/9904477] [INSPIRE].

[62] S.V. Mikhailov and A.V. Radyushkin, Structure of Two Loop Evolution Kernels and Evolution of the Pion Wave Function in $\phi^{3}$ in Six-dimensions and QCD, Nucl. Phys. B 273 (1986) 297 [INSPIRE].

[63] D. Mueller, Conformal constraints and the evolution of the nonsinglet meson distribution amplitude, Phys. Rev. D 49 (1994) 2525 [InSPIRE].

[64] D. Mueller, The evolution of the pion distribution amplitude in next-to-leading-order, Phys. Rev. D 51 (1995) 3855 [hep-ph/9411338] [INSPIRE].

[65] Y. Grossman, M. König and M. Neubert, Exclusive Radiative Decays of $W$ and $Z$ Bosons in QCD Factorization, JHEP 04 (2015) 101 [arXiv: 1501.06569] [INSPIRE].

[66] B.L. Ioffe and A.V. Smilga, Nucleon Magnetic Moments and Magnetic Properties of Vacuum in QCD, Nucl. Phys. B 232 (1984) 109 [INSPIRE].

[67] M. König and M. Neubert, Exclusive Radiative Higgs Decays as Probes of Light-Quark Yukawa Couplings, JHEP 08 (2015) 012 [arXiv: 1505.03870] [INSPIRE].

[68] M.A. Shifman and M.I. Vysotsky, Form-Factors Of Heavy Mesons In QCD, Nucl. Phys. B 186 (1981) 475 [INSPIRE].

[69] X.-P. Wang and D. Yang, The leading twist light-cone distribution amplitudes for the $S$-wave and P-wave quarkonia and their applications in single quarkonium exclusive productions, JHEP 06 (2014) 121 [arXiv:1401.0122] [INSPIRE]. 
[70] G.P. Lepage and S.J. Brodsky, Exclusive Processes in Quantum Chromodynamics: Evolution Equations for Hadronic Wave Functions and the Form-Factors of Mesons, Phys. Lett. B 87 (1979) 359 [INSPIRE].

[71] K.G. Chetyrkin, Quark mass anomalous dimension to $O\left(\alpha S^{4}\right)$, Phys. Lett. B 404 (1997) 161 [hep-ph/9703278] [INSPIRE].

[72] J.A.M. Vermaseren, S.A. Larin and T. van Ritbergen, The four loop quark mass anomalous dimension and the invariant quark mass, Phys. Lett. B 405 (1997) 327 [hep-ph/9703284] [INSPIRE].

[73] Y.-M. Wang and Y.-L. Shen, Perturbative Corrections to $\Lambda_{b} \rightarrow \Lambda$ Form Factors from QCD Light-Cone Sum Rules, JHEP 02 (2016) 179 [arXiv:1511.09036] [INSPIRE].

[74] S.V. Mikhailov and A.A. Vladimirov, ERBL and DGLAP kernels for transversity distributions. Two-loop calculations in covariant gauge, Phys. Lett. B 671 (2009) 111 [arXiv:0810.1647] [INSPIRE].

[75] A.V. Belitsky, A. Freund and D. Mueller, NLO evolution kernels for skewed transversity distributions, Phys. Lett. B 493 (2000) 341 [hep-ph/0008005] [INSPIRE].

[76] V.M. Braun, A.N. Manashov, S. Moch and M. Strohmaier, Three-loop evolution equation for flavor-nonsinglet operators in off-forward kinematics, JHEP 06 (2017) 037 [arXiv: 1703.09532] [INSPIRE].

[77] V.L. Chernyak and A.R. Zhitnitsky, Exclusive Decays of Heavy Mesons, Nucl. Phys. B 201 (1982) 492 [Erratum ibid. B 214 (1983) 547] [INSPIRE].

[78] A.P. Bakulev, S.V. Mikhailov and N.G. Stefanis, QCD based pion distribution amplitudes confronting experimental data, Phys. Lett. B 508 (2001) 279 [Erratum ibid. B 590 (2004) 309] [hep-ph/0103119] [INSPIRE].

[79] V.L. Chernyak, Selected topics in $e^{+} e^{-}$collisions, Nucl. Phys. Proc. Suppl. 162 (2006) 161 [hep-ph/0605327] [INSPIRE].

[80] A. Khodjamirian, T. Mannel, N. Offen and Y.M. Wang, $B \rightarrow \pi \ell \nu_{l}$ Width and $\left|V_{u b}\right|$ from QCD Light-Cone Sum Rules, Phys. Rev. D 83 (2011) 094031 [arXiv:1103.2655] [InSPIRE].

[81] S.J. Brodsky and G.F. de Teramond, Light-Front Dynamics and AdS/QCD Correspondence: The Pion Form Factor in the Space- and Time-Like Regions, Phys. Rev. D 77 (2008) 056007 [arXiv: 0707.3859] [INSPIRE].

[82] I.C. Cloët, L. Chang, C.D. Roberts, S.M. Schmidt and P.C. Tandy, Pion distribution amplitude from lattice-QCD, Phys. Rev. Lett. 111 (2013) 092001 [arXiv:1306.2645] [INSPIRE].

[83] V.M. Braun et al., Second Moment of the Pion Light-cone Distribution Amplitude from Lattice QCD, Phys. Rev. D 92 (2015) 014504 [arXiv:1503.03656] [InSPIRE].

[84] V.A. Novikov, M.A. Shifman, A.I. Vainshtein, M.B. Voloshin and V.I. Zakharov, Use and Misuse of QCD Sum Rules, Factorization and Related Topics, Nucl. Phys. B 237 (1984) 525 [INSPIRE].

[85] P. Ball, V.M. Braun and A. Lenz, Higher-twist distribution amplitudes of the $K$ meson in QCD, JHEP 05 (2006) 004 [hep-ph/0603063] [INSPIRE]. 
[86] J. Rohrwild, Determination of the magnetic susceptibility of the quark condensate using radiative heavy meson decays, JHEP 09 (2007) 073 [arXiv:0708.1405] [INSPIRE].

[87] A. Vainshtein, Perturbative and nonperturbative renormalization of anomalous quark triangles, Phys. Lett. B 569 (2003) 187 [hep-ph/0212231] [INSPIRE].

[88] Y.-M. Wang and Y.-L. Shen, QCD corrections to $B \rightarrow \pi$ form factors from light-cone sum rules, Nucl. Phys. B 898 (2015) 563 [arXiv:1506.00667] [INSPIRE].

[89] Y.-M. Wang, Y.-B. Wei, Y.-L. Shen and C.-D. Lü, Perturbative corrections to $B \rightarrow D$ form factors in QCD, JHEP 06 (2017) 062 [arXiv:1701.06810] [INSPIRE].

[90] A. Khodjamirian, T. Mannel and N. Offen, Form-factors from light-cone sum rules with B-meson distribution amplitudes, Phys. Rev. D 75 (2007) 054013 [hep-ph/0611193] [INSPIRE].

[91] CLEO collaboration, J. Gronberg et al., Measurements of the meson-photon transition form-factors of light pseudoscalar mesons at large momentum transfer, Phys. Rev. D 57 (1998) 33 [hep-ex/9707031] [INSPIRE].

[92] RQCD collaboration, G.S. Bali et al., Second moment of the pion distribution amplitude with the momentum smearing technique, Phys. Lett. B 774 (2017) 91 [arXiv:1705.10236] [INSPIRE].

[93] M. Unverzagt, Light meson physics with Crystal Ball/MAMI and at BES-III, J. Phys. Conf. Ser. 349 (2012) 012015 [INSPIRE].

[94] H.-n. Li and S. Mishima, Pion transition form factor in k(T) factorization, Phys. Rev. D 80 (2009) 074024 [arXiv:0907.0166] [INSPIRE].

[95] BABAR collaboration, J.P. Lees et al., Measurement of the $\gamma \gamma * \rightarrow \eta_{c}$ transition form factor, Phys. Rev. D 81 (2010) 052010 [arXiv:1002.3000] [INSPIRE].

[96] E.G. Floratos, D.A. Ross and C.T. Sachrajda, Higher Order Effects in Asymptotically Free Gauge Theories: The Anomalous Dimensions of Wilson Operators, Nucl. Phys. B 129 (1977) 66 [Erratum ibid. B 139 (1978) 545] [INSPIRE].

[97] A. Gonzalez-Arroyo, C. Lopez and F.J. Yndurain, Second Order Contributions to the Structure Functions in Deep Inelastic Scattering. 1. Theoretical Calculations, Nucl. Phys. B 153 (1979) 161 [INSPIRE].

[98] A.V. Belitsky and A.V. Radyushkin, Unraveling hadron structure with generalized parton distributions, Phys. Rept. 418 (2005) 1 [hep-ph/0504030] [INSPIRE].

[99] W. Vogelsang, Next-to-leading order evolution of transversity distributions and Soffer's inequality, Phys. Rev. D 57 (1998) 1886 [hep-ph/9706511] [INSPIRE].

[100] A. Hayashigaki, Y. Kanazawa and Y. Koike, Next-to-leading order $Q^{2}$ evolution of the transversity distribution $h_{1}\left(x, Q^{2}\right)$, Phys. Rev. D 56 (1997) 7350 [hep-ph/9707208] [INSPIRE]. 